\title{
Are Cooperative Banks Better Equipped to Weather Financial Crisis than their Commercial Counterparts? Evidence from the Italian Banking Sector before and during the Credit Crisis
}

\author{
Mitja Stefancic \\ Via Frescobaldi, 31 - 34148 Trieste, Italy \\ mitja.s@hotmail.it
}

\begin{abstract}
Background and Purpose: The aim of this paper is to empirically investigate the performance of different types of Italian banks before and during the recent credit crisis with an emphasis on the behaviour of cooperative banks. It is well established in theory that cooperative banks follow more conservative business strategies and care more for stakeholders in comparison to commercial banks. On this background, the paper tries to show the empirical effects of those characteristics on the cooperative bank's performance during financial distress compared to commercial banks. In fact, the paper can prove that Italian cooperative banks were less exposed to the shocks of the crisis and showed a better performance.

Methodology: In order to assess whether cooperative banks performed differently at all from commercial banks during the 2005-2012 period, return on average assets (ROAA), cost efficiency and loan quality have been investigated by means of a sample of 594 Italian banks, pooled OLS and (when possible) a fixed effects estimator.

Results: Overall, Italian cooperative banks performed better than other Italian banks during the financial crisis. The quality of loans deteriorated less in these banks than in others, while no significant differences have been observed in terms of ROAA and cost efficiency between these and other banks.

Conclusion: My paper provides empirical evidence for a well established theoretically derived hypothesis: Italian cooperative banks operate differently than standard commercial banks which is especially noticeable during times of crisis. The fact empirically demonstrated that different banking models have shown different reactions to the financial crisis and economic downturn has important policy implications. Due to both characteristics of cooperative banks and severe limitations in the financial policies by the Italian government during the credit crisis an ironical pattern has emerged: While Italian cooperative banks were less exposed to the shocks of the crisis, they would have been less able to adjust to them since the financial rescue program was designed primarily for commercial banks.
\end{abstract}

Keywords: cooperative banks, bank performance, bank efficiency, bank soundness, credit crisis

\section{Introduction}

The aim of this paper is to investigate the performance of Italian banks before and during the recent financial crisis. Specifically, the paper centres on two types of banks: commercial banks, namely privately owned banks that provide services both to the general public and to private firms, and cooperative banks, namely those with a per capita voting mechanism that provide services mainly to cooperative members, households and small enterprises. The last credit crisis reminded us that a sound banking system builds on profitable and well-capitalised banks that 
are able to operate efficiently while successfully managing their risks. Recent scholarly research on commercial banks (Dewatripont and Freixas, 2012; Fahlenbrach et al., 2012; Vallascas and Hagendorff, 2013; Mohsni and Otchere, 2015) and the evidence from the credit crisis suggest that many commercial banks were not satisfying these criteria prior to the crisis. Since they are established with the purpose of maximising profits, commercial banks' primary goal is the creation of profits or, more exactly, the maximisation of shareholder value. This goal, the small share of equity in banks' liabilities, the deposit insurance and the implicit state guarantee due to the systemic role of the banks, may motivate managers in commercial banks to assume larger risks, particularly in cases where they are owned by influential private institutional owners (e.g. Laeven and Levine, 2006).

On the other hand, pre-crisis scholarly research on both Italian and European cooperative banks indicates that cooperative banks are, on average, less profitable in "normal" periods but also more stable due to higher solvency ratios (Hesse and Cihak, 2007; Gutierrez, 2008). As a result of their embeddedness in local economic systems, their institutional legacies and mutualistic values, cooperative banks tend to adopt conservative business strategies and stakeholder value maximisation policies in comparison to commercial banks (Ferri, 2008; Stefancic, 2011; Manetti and Bagnoli, 2013; Chiaramonte et al., 2013). They are customer-oriented, and particularly efficient at maintaining long-lasting relationships with their members and customers. In other words, these banks are particularly strong at relationship banking, a strategy that enables banks to make informed decisions on the provision of loans and financial services as a result of in-depth knowledge of customers' business. Relationship banking generates a number of advantages, such as proximity to customers (Boot, 1999; Boot and Thakor, 2000; Cesarini, 2003; Di Salvo et al., 2004; Oliver Wyman, 2014), which may contribute positively to the quality of these banks' loans. In Italy, cooperative banks can also rely on a welldeveloped commercial network with important historical roots and market advantages (Angelini and Cetorelli, 2003; Finocchiaro, 2007; Leonardi, 2009), which may help to ease their access to information about customers. These specifics may make cooperative banks less vulnerable to shocks to the system, as was the case in the last credit crisis.

In order to assess whether the behaviour of Italian cooperative banks had differed significantly from that of commercial banks especially during the crisis, a model is estimated in which return on average assets (ROAA), the cost-to-income ratio and a measure of loan quality are regressed on a number of indicator (dummy) variables indicating the bank type (cooperative banks, people's banks, savings banks, commercial banks) and a set of control variables, as suggested in the literature (Ferri et al.,
2010). The latter variables aim to account for bank size, asset quality and type of activity (e.g. non-interest income), capital ratios and liquidity. The analysis is based on an unbalanced panel of 594 Italian banks during 2005-2012. The focus is on the differences between the behaviour of cooperative banks and other banks, and on the differential impact of the credit crisis on these banks: here, the concept 'behaviour' is understood as the result of a number of factors including not only the strategy and organizational structure of a bank, but also its fundamental values (for instance mutualism in the case of cooperative banks). Arguably, such differences would suggest that, regardless of the fact that they compete with other banks, cooperative banks can be indeed considered to be a specific type of financial institution. This would necessitate an adjustment in the regulatory rules that apply to banks. The present paper may thus be viewed as a contribution towards a constructive discussion on market regulation policies for banks.

The present analysis focuses on the Italian banking sector. Italy is one of the most important European economies. Indeed, it is the third-largest Euro zone economy after Germany and France. According to a recent assessment by the IMF (2013, p. 9), 'banks account for almost $85 \%$ of total financial sector assets. At end-2012, there were 706 banks with total assets of about $220 \%$ of GDP, of which 169 were part of 75 banking groups'. Cooperative banks are particularly important as for instance they tend to mitigate credit-rationing to SMEs and specific market segments in Italy (Gutierrez, 2008). In addition to that, the relevance of the present paper is underlined by the fact that Italian cooperative banks are currently subject to important reforms of which the final outcomes are still not so clear (Stefancic, 2014; The Economist, 2015). A focus on Italy is a promising approach since cooperative banks have proved to be essential here for the development of culturally and economically rich local economies that could successfully adapt to the process of globalization.

Although the Italian banking system underwent a process of restructuring and consolidation in the 1990s, cooperative banks still play an important role today. For example, in 1999 there were a total of 580 cooperative banks operating in Italy; these banks employed 70,636 employees in 7,067 branches, and held approximately EUR 287,000 million in assets. By the year 2009, this number had fallen to 459 cooperative banks, employing 62,755 employees in a slightly larger number of branches (7,311). The value of cooperative banks' assets had nearly doubled during the same period (to EUR 512,000 million). For comparison, in 1999 the number of commercial banks (at 296) was much lower than the number of cooperative banks and had increased slightly by 2009 (to 329 banks). Commercial banks are bigger institutions; in 1999, they operated in 20,067 branches, employing 270,675 employees and holding EUR 1,432,994 million in assets. 
These numbers are larger than those for the cooperative banks, and increased during the 1999-2009 period (to 26,724 branches, 259,820 employees and EUR 2,942,195 million in assets $)^{1}$.

This paper is structured as follows. Section 2 provides a discussion on the specifics of Italian cooperative banks based on the literature review. Building on these specifics and on the existing evidence on bank performance during the last credit crisis, the main hypotheses to be tested in this paper are derived. Section 3 provides an outline of the sample and methodology used for the analysis. The main empirical analysis and results are discussed in Section 4. Section 5 concludes by commenting on the results and deriving implications for financial policy. Results provided in this paper and relevant policy suggestions should be of particular interest to scholars and policy makers focusing on cooperative and mutual banks or on cooperative enterprises more generally.

\section{Literature review and hypotheses}

Empirical evidence on bank performance in relation to different ownership types, in particular the results for the Italian market, and the European market as a whole, is mixed. Nevertheless, based on the papers surveyed below, it can safely be argued that, in most cases, cooperative banks do not lag behind their commercial counterparts (by way of exception, a few studies show the superiority of commercial over cooperative banks in terms of profitability). To start with, Ianotta et al. (2007) compare the behaviour of large banks from 15 European countries during the 1994-2004 period, and find that mutual and government-owned banks have lower levels of profitability. Nonetheless, they also find that mutual banks better manage their loan portfolios and have lower asset risk than commercial banks. Goddard et al. (2004) focus on the profitability of 665 banks from Denmark, France, Germany, Italy, Spain and the UK. On the basis of their results there appears to be no convincing relationship between ownership type, size and bank performance, except in Germany: German savings banks and cooperative banks appear to have been less profitable than German commercial banks during the 1990s. Ferri et al. (2010) use a panel of more than 300 banks from several European countries to study different types of banks in the period 1994-2008. They find no significant differences in the profitability of different bank types, whereas, in terms of cost efficiency, cooperative banks slightly outperform commercial banks.

Another set of papers focus on Italian banks alone. With reference to the loan-granting process of Italian banks for the period 2000-2006, Mattarocci and Gibilaro (2008) show that, from an operational point of view, small financial intermediaries such as the Italian cooperative credit banks have a better-quality loan-granting process. At the same time, these banks are able to implement more efficient recovery processes. Girardone et al. (2004) investigate the determinants of Italian banks' cost efficiency during the 1993-1996 period. They show that inefficiencies are inversely correlated with capital strength; on the other hand, they are positively related to the level of nonperforming loans in the balance sheet. With reference to estimates of the Bank of Italy and some pre-crisis research, Gutierrez (2008) argues that cooperative banks are more cost efficient yet their profitability is lower in comparison to commercial banks despite the fact that they seem to enjoy a higher degree of monopoly power (as showed by the estimated H-statistic for different types of institutions).

Bonanno (2012) evaluates the efficiency of Italian banks by means of a stochastic frontier approach. With reference to a sample for the 2006-2010 period, the study shows that a sharp reduction in bank efficiency occurred in the year 2008. Despite that, cooperative credit banks performed better than non-cooperative counterparts over the 20062010 period. Using a similar analytical method, Aiello and Bonanno (2013) evaluate the cost and profit efficiency of Italian banks over the 2006-2011 period. Their results indicate that Italian banks generally perform well in terms of cost efficiency and profitability, and that banks are also quite stable over time. However, they acknowledge high heterogeneity in their results - something that is relevant to our discussion: differences are significant when banks are classified either by size (efficiency tends to decrease with size) or legal type (cooperatives tend to outperform other types of banks). Manetti and Bagnoli (2013) focus on the concept of efficiency in cooperative banks from Tuscany, with reference to the mutuality and sustainability of their business. By re-elaborating indicators such as the Value Added and the Cost to Income ratio, the authors show that the performance of such banks for the years 2009 and 2010 is close to the average performance of standard commercial Italian banks, and that the banche di credito cooperativo are both efficient and mission-oriented.

Consequently, based on the specifics of the cooperative banks' operations and business strategies, the following hypothesis is developed:

H1: To achieve their institutional goal of long-term financial stability and economic development of their reference territory, cooperative banks pursue more conservative policies than commercial banks, resulting in lower riskiness of bank operations, yet also in at least equal levels of profitability.

As argued above, in normal times cooperative banks tend to apply safer business strategies and take on lower risks than commercial banks, and may as a result be less

${ }^{1}$ For an overview of comparable data on European cooperative banks at an aggregate level refer to Groeneveld (2015). 
exposed to the effects of a financial crisis. As explicated by $\mathrm{H} 1$, this is related to the institutional goals of cooperative credit banks to promote safe investments and sound operations as well as encourage strong relations with members and customers ${ }^{2}$. In line with this theoretical expectation, the European Association of Cooperative Banks (EACB) notes that 'more than $95 \%$ of write downs registered worldwide were due to commercial banks and some public banks; the cost in terms of loan loss provisions seems more equally distributed. Recapitalisation (in particular state aid) was also massively directed towards commercial banks and some public banks. Cooperative banks have therefore had little responsibility for the direct costs of the crisis, despite their heavy weight in the economy, with about $20 \%$ in terms of market share' (2010, p. 8). The last credit crisis reminded us that a sound banking system builds on profitable and well-capitalised banks that are able to operate efficiently while successfully managing (controlling) their risk exposure. Recent papers on commercial banks and the evidence from the crisis (Dewatripont and Freixas, 2012; Fahlenbrach et al., 2012; Vallascas and Hagendorff, 2013; Mohsni and Otchere, 2015) suggest that many commercial banks under pressure for high profitability, were not satisfying these criteria prior to the crisis ${ }^{3}$.

Moreover, the last credit crisis demonstrated the degree of interconnectedness among financial intermediaries worldwide, has been matched by a decrease in trust towards banking intermediaries and among banks themselves. This, in turn, stresses the importance of trustworthy relations between banks and between them and their customers, particularly in the event of shocks such as the last crisis. Since trust is a distinctive feature of many cooperative banks, such banks should have a competitive advantage over commercial and other banks in developing and maintaining long-lasting relationships with their borrowers and, most importantly, their depositors. All of the above should imply that cooperative banks will perform better than other banks during crisis periods. However, cooperative banks are, in a way, "forced" to maintain close relationships with local communities and economic agents, as shown by the association between these banks and the local economy (Gallo et al., 2011), which often prevents them from reaching other markets. A careful review of the arguments developed in the mentioned papers (e.g. Manetti and Bagnoli, 2013) suggests that cooperative banks in Italy are still heavily dependent on the relationship with the local economic systems.

Consequently, while being able to preserve their sources of financing, these banks might be also be inclined to support their customers even when it will reduce their profits in the short run to do so. Moreover, the state aid provided during the crisis was directed primarily at commercial banks and, in some countries, at some large public banks. As in other parts of Europe, cooperative credit banks in Italy were largely excluded from such aid (EACB, 2010). Furthermore, communitarian frameworks aimed at helping banks have been designed with mediumsized and large commercial banks in mind. According to a report by Morgan Stanley Europe (2012), Italian banks benefitted substantially from operations such as the long term refinancing operations (LTRO), but it was mainly commercial banks and large banche popolari that received help. These last few factors imply that - while less exposed to the crisis in the first place - the cooperative banks would have been less able to react to the crisis shock. However, one could expect that this latter effect had a smaller impact on cooperative banks than the former (positive) effect. Consequently, I suggest to state the second and third hypothesis as follows:

H2: The credit crisis led to a significant decrease in profitability, cost efficiency and loan quality in all types of banks - despite some types of banks having been less exposed to the crisis than others.

H3: Given the greater stability of their operations, cooperative banks were less effected by the crisis than commercial banks.

\section{Data and methodology}

\subsection{Data}

The empirical analysis in this paper is based on the onbalance-sheet data from a sample of 594 banks operating in Italy during 2005-2012. This sample represents a large share of the entire Italian banking system (more than $80 \%$ of all banks). In 2012, for example, a total of 724 banks were operating in the Italian market (Tidona Comunicazione, 2013). The banks included in the sample are of different types, comprising 355 cooperative banks, 49 people's banks, 35 savings banks and 155 commercial

\footnotetext{
${ }^{2}$ For more information about the institutional goals of cooperative banks, please refer to the Chart of Values of Cooperative Banks (specifically, the 'Carta dei Valori' for Italian banks of this type). See also Stefancic (2011 and 2014).

${ }^{3}$ For example, Fahlenbrach et al. (2012) find that a bank's risk culture influences the outcomes of its operations and business. In particular, commercial banks that performed poorly in the past (i.e. during the 1998 crisis), rely more on short-term funding and had low risk management, appear to have been also less resilient to the recent financial crisis, facing a higher probability of failure. Vallascas and Hagendorff (2013) evaluate risk sensitivity and capital requirements of banks by considering a sample of large international banks for the period 2000-2010. They find that low-risk sensitivity of banks may prevent them from adequately withstanding adverse shocks. A review of the above and other relevant studies is present in Stefancic (2014).
} 
banks. The for-profit banks (commercial and savings banks $)^{4}$ represent slightly less than $32 \%$ of the sample, while the non-profit banks (cooperative and people's banks) represent the majority of the banks in the sample. Financial information on the banks was obtained from the BankScope database provided by Bureau van Dyke. The data refer to the eight-year period 2005-2012, which includes a period of severe financial distress (particularly the year 2008 onwards), and the Euro crisis of 2011. Thus, the data include periods of severe shocks to the banking system (e.g. Quagliariello, 2008; Bank of England, 2009; Freixas, 2009).

A potential problem in the sample selection relates to the definition of cooperative banks. The definition is not straightforward. Some studies classify all banks with a per capita voting mechanism, for example mutual and rural banks, as cooperative banks (Battistin et al., 2006 and 2012). If taken together, these banks account for approximately $30 \%$ of both loans and deposits in the Italian banking system. However, significant differences exist between such categories of banks. In the construction of the database, the classification from the Italian Banking Act (Testo unico delle leggi in materia bancaria e creditizia) and insights from Gutierrez (2008) were followed, differentiating between the cooperative banks (banche di credito cooperativo) and the people's banks (banche popolari).

\subsection{Empirical model}

The evaluation of the performance of cooperative and other Italian banks is based on three dependent variables: (i) a variable measuring bank profitability, namely the return on average assets (ROAA); (ii) a variable measuring cost efficiency (COST_EFFICIENCY); (iii) a variable measuring the soundness of bank loans (LOAN_ PROVISIONS). As noted by Ferri et al. (2010) among others, it is important to consider other measures than profitability in order to account for the distinct objective function cooperative banks have in comparison to other banks. The ROAA is defined as net income divided by total average assets, and is useful for assessing profitability. COST_EFFICIENCY is defined as the ratio of a bank's costs to its total revenues (income), i.e. the cost-to-income ratio, measured in percentage terms. It is commonly used in studies on bank efficiency (Ferri et al., 2010; Manetti and Bagnoli, 2013). LOAN_PROVISIONS stands for a bank's loan loss provisions as a share of the total amount of gross loans. These definitions follow the literature and the definitions suggested by the BankScope database. In relation to bank profitability, other measures could be considered, such as the return on equity (ROE) $)^{5}$.

The main explanatory variables are dummy variables capturing the type of bank: COOP takes the value 1 for cooperative banks, and 0 otherwise; POP takes the value 1 for popular banks, and 0 otherwise; finally, in selected specifications, SAVING takes the value 1 for savings banks, and 0 otherwise. As an alternative, in robustness checks the variable NON-PROFIT, which denotes both cooperative and people's banks (with commercial and savings banks being the reference group), is used (see Appendices). In order to test $\mathrm{H} 3$ which relates to differences in the banks' behaviour during the crisis, the interaction term COOP*CRISIS is introduced, in which the variable CRISIS is a dummy taking the value 1 in years 2009 onwards, and 0 otherwise, with COOP defined as above. The choice of 2009 as the starting year for the crisis period allows for the fact that the US crisis probably hit the Italian market with some delay; this is also supported by the analysis of the time dummy variables, where the first significant declines in bank performance can be seen in the year 2009. For robustness, the main regressions are re-estimated using the year 2008 as the starting year for the crisis period.

The choice of control variables was guided mostly by the existing studies in the field (in particular Ferri et al., 2010) and by data availability. A set of controls is introduced, capturing differences in bank size, asset quality, activity type, capital ratios, etc. Following Ferri et al. (2010) and other studies, bank size is controlled for by means of the logarithm of total bank assets (InTOTALASSETS). Bank capitalisation is measured by the share of equity in total bank assets (EQUITY_ASSETS) and, alternatively, by a regulatory measure of banks' capitalisation (TOTALCAPITAL_RATIO). To capture different types of bank activity and the structure of bank assets, further control is posed on the share of customer (non-bank) deposits in assets (CUSTOMERDEP_ASSETS), the share of loans in total bank assets (NETLOANS_ASSETS), the share of liquid assets in total assets (LIQUID_ASSETS), and the share of non-interest income in total bank revenues (NONINTEREST). These variables are measured in percentage terms. All of the regressions include time dummies and, when specified, bank fixed effects.

\footnotetext{
${ }^{4}$ As a result of the banking reforms and consolidation process in Italy, current savings banks operate as commercial banks or very similarly.

${ }^{5}$ While some studies and policy makers adopt this ratio as the most reliable indicator of profitability, others have criticised its use and prefer to rely on other indicators (Karr, 2005; Tumpel-Gugerell, 2005; De Bonis, 2008, pp. 114-116; Ferri et al. 2010), such as the ROA. As observed by Dietrich and Wanzenried $(2009,2011)$, the ROAA provides a good approximation of bank profitability, and reflects the ability of a bank's management to generate profits from the bank's assets. This measure should be preferred to ROE as the latter does not capture financial leverage and the risks associated with it.
} 


\section{Empirical analysis}

\subsection{Descriptive statistics}

First are presented the descriptive statistics across the entire period of analysis, separately for each type of bank. For the purpose of the empirical analysis, outliers were transformed by winsorising the lower and highest $1 \%$ of values for the non-dummy variables used in the analysis. Winsorisation is a way of transforming the outliers without discarding them and therefore without losing information ${ }^{6}$. Cooperative banks are much smaller than the commercial banks and even the other types of bank. Size differences between the cooperative and other banks do, of course, pose a problem for the analysis, as small and large banks may differ in many other characteristics that cannot be adequately controlled for in the model.

Differences between banks are observable on the basis of their median values of the selected variables. All of them are expressed as percentages in the above table, except for the total assets, which are in thousands Euros. Most variables have a slightly asymmetric distribution at least for one bank type, therefore median values provide a better measure of central tendency to make comparisons than the mean ones.

The dependent variables are commented first. Cooperative credit banks have the highest return on average assets $(0.56 \%)$, meaning that they are the most profitable bank type according to this metric, followed by savings banks $(0.53 \%)$. Commercial banks are the least profitable according to ROAA $(0.45 \%)$. With regards to loan losses, people's banks are those that perform worse $(0.66 \%)$, followed by commercial banks $(0.61 \%)$. Savings banks have lower levels of loan losses, while cooperative credit banks are those that show the best results for the considered period $(0.52 \%)$. That is to say, they are the best type of banks when the soundness of bank loans is considered. Finally, cooperative credit banks exceed other banks in terms of the cost to income ratio with a median value of $66.67 \%$ compared to $62.23 \%$ of commercial banks (people's and savings banks are in between).

Next, the discussion centres on differences in the explanatory variables. In terms of total assets, people's banks are those with the highest median value $(3,973,550,000 €)$, and are followed by commercial $(2,447,450,000 €)$ and savings banks $(2,427,100,000 €)$. Cooperative credit banks have the lowest median level of assets $(284,300,000 €)$. Total assets have a very skewed distribution, therefore a logarithmical transformation has been used in the models.
With regards to customer deposits, the highest values are registered for savings and people's banks $(50.56 \%$ and $50.28 \%$, respectively), followed by cooperative banks (49.87\%) and, eventually, commercial banks (40.31\%). Structural differences are observable also with respect to the share of net loans on total assets: the median values for the four bank types vary between the $76.96 \%$ of savings banks and $67.47 \%$ of commercial banks, with people's banks $(73.27 \%)$ and cooperative credit banks $(68.72 \%)$ in between.

Moving to liquidity, measured as the share of liquid assets on customer and short term funding, it can be observed that commercial banks (14.82\%) are the most liquid type, whereas cooperative credit banks (8.51\%) are the least liquid. Savings banks $(9.16 \%)$ and people's banks $(11.79 \%)$ are in between. With regards to noninterest income as a share of revenues, the share is the highest for commercial banks $(35.44 \%)$, and the lowest for cooperative credit banks (22.91\%) - differences in this respect are clear. Finally, in terms of bank capitalisation, cooperative credit banks are the most capitalised as showed by the highest values for equity as a share of total assets $(10.86 \%)$, followed by people's banks $(9.80 \%)$, commercial banks $(8.01 \%)$ and savings banks $(8.07 \%)$. Similarly, cooperative credit banks have the highest total capital ratio (15.61\%), whereas savings banks are the least capitalised with a total capital ratio of $10.99 \%$.

\subsection{Statistical modelling}

\subsubsection{Methodology}

The descriptive statistics presented above provide an idea of the differences between the cooperative and other banks, as well as an indication of how well each of these banks' groups reacted to the crisis in comparison to other banks. Making inferences based on the averages is not appropriate, however, as the differences between the different types of banks may be due to differences in other bank characteristics than bank type, such as size, capitalisation, etc. To address this, an empirical analysis is carried out in which selected measures of bank performance (in terms of profitability, cost efficiency and loan quality) are regressed on an indicator variable for the type of bank (cooperative, savings, people's or commercial) and a set of control variables. The choice of estimator is mainly influenced by the nature of our main explanatory variable, which does not vary over time. Therefore, in most of the cases the study relies on the pooled OLS estimator with standard errors clustered at the level of bank type, or alternatively at the level of the individual bank to correct for serial correlation

\footnotetext{
${ }^{6}$ In the present case, a $98 \%$ winsorisation has been applied, this is, for each variable, data smaller the first percentile have been set at the value of the first percentile and data larger than the 99th percentile have been set at the value of the 99th percentile. Such an approach is common in the finance literature.
} 
Table 1: Descriptive statistics (Source: Author's calculations)

\begin{tabular}{|c|c|c|c|c|c|c|c|c|c|c|c|c|}
\hline \multirow{6}{*}{ 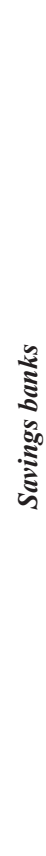 } & $\begin{array}{l}\overline{0} \\
0 \\
\vdots\end{array}$ & $\vec{\sim}$ & $\vec{\sim}$ & $\vec{\nabla}$ & $\vec{\sim}$ & $\vec{\sim}$ & $\stackrel{\bullet}{\sim}$ & $\vec{\sim}$ & ปี & $\overrightarrow{\widetilde{0}}$ & $\overrightarrow{0}$ & $\vec{\nabla}$ \\
\hline & $\begin{array}{l}\Xi \\
\Xi \\
\Xi\end{array}$ & $\begin{array}{l}\tilde{D} \\
\stackrel{n}{0}\end{array}$ & 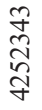 & $\begin{array}{l}\text { 我 } \\
\stackrel{0}{0} \\
i\end{array}$ & $\begin{array}{l}\bar{\sigma} \\
\bar{\Xi}\end{array}$ & $\frac{\stackrel{8}{\circ}}{\stackrel{8}{\circ}}$ & $\begin{array}{l}\vec{J} \\
\vec{d}\end{array}$ & $\frac{8}{\stackrel{\circ}{\circ}}$ & $\begin{array}{l}\text { 年 } \\
\infty \\
= \\
=\end{array}$ & 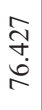 & $\begin{array}{l}\vec{\infty} \\
\underset{\infty}{\infty}\end{array}$ & $\begin{array}{l}\hat{\varrho} \\
\dot{ \pm} \\
\pm\end{array}$ \\
\hline & 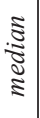 & $\stackrel{n}{n}$ & 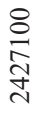 & $\begin{array}{l}\hat{0} \\
n \\
i \\
i\end{array}$ & $\frac{d}{\sigma^{\circ}}$ & $\stackrel{m}{\stackrel{m}{m}}$ & $\vec{\sigma}$ & $\begin{array}{l}n \\
n \\
n\end{array}$ & ڤ్ & 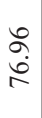 & $\hat{\infty}$ & $\begin{array}{l}\stackrel{\Im}{尺} \\
\dot{\Xi}\end{array}$ \\
\hline & 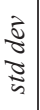 & $\begin{array}{l}\vec{\infty} \\
n \\
? \\
0\end{array}$ & 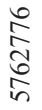 & $\begin{array}{l}8 \\
\text { ọ } \\
\sigma\end{array}$ & $\begin{array}{l}\vec{b} \\
\stackrel{\infty}{0}\end{array}$ & $\stackrel{\stackrel{N}{r}}{\stackrel{r}{r}}$ & $\frac{i}{\infty}$ & 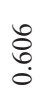 & 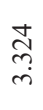 & $\begin{array}{l}\infty \\
\underset{\infty}{\infty} \\
\infty\end{array}$ & 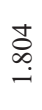 & 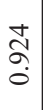 \\
\hline & $\stackrel{\Xi}{\Xi}$ & $\stackrel{\text { aे }}{\text { ì }}$ & $\begin{array}{l}0 \\
+ \\
0 \\
8 \\
8 \\
\dot{+}\end{array}$ & $\begin{array}{l}\mathscr{\infty} \\
\stackrel{\infty}{+} \\
\dot{\nabla}\end{array}$ & $\begin{array}{l}\text { mे } \\
\stackrel{f}{f}\end{array}$ & $\begin{array}{l}n \\
n \\
n\end{array}$ & n̊ & $\begin{array}{l}\hat{2} \\
\infty \\
+\end{array}$ & ले & $\begin{array}{l}\text { ñ } \\
\text { }\end{array}$ & $\begin{array}{l}\tilde{\sigma} \\
\dot{J} \\
-\end{array}$ & $\begin{array}{l}0 \\
\\
\end{array}$ \\
\hline & $\stackrel{\Xi}{\Xi}$ & $\begin{array}{l}\overrightarrow{0} \\
\stackrel{n}{?} \\
\dot{\varphi}\end{array}$ & $\frac{\substack{0\\
}}{i n}$ & $\begin{array}{l}\hat{\jmath} \\
\text { ஸे } \\
\infty \\
\sim\end{array}$ & กิ & $\begin{array}{c}2 \\
\infty \\
\infty \\
1\end{array}$ & $\frac{\tilde{y}}{\stackrel{\gamma}{+}}$ & 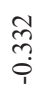 & $\frac{n}{r}$ & $\frac{5}{a}$ & $\begin{array}{l}\text { है } \\
\text { r }\end{array}$ & $\begin{array}{l}n \\
\tilde{n} \\
n \\
n\end{array}$ \\
\hline \multirow{7}{*}{ 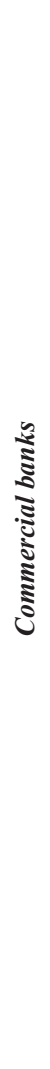 } & $\begin{array}{l}\overrightarrow{n े} \\
0 \\
\vdots\end{array}$ & ஜ̊ & $\stackrel{\infty}{8}$ & $\frac{\pi}{a}$ & $\stackrel{\infty}{8}$ & ă & م) & $\underset{\infty}{\stackrel{2}{\infty}}$ & $\frac{N}{\infty}$ & $\frac{n}{a}$ & $\stackrel{\infty}{8}$ & $\stackrel{\infty}{0}$ \\
\hline & $\begin{array}{l}\Xi \\
\Xi \\
\cong\end{array}$ & $\vec{i}$ & $\begin{array}{l}\hat{0} \\
+ \\
0 \\
\text { ஸे } \\
\text {. }\end{array}$ & $\underset{n}{\frac{n}{n}}$ & $\begin{array}{l}\hat{\sigma} \\
\dot{d} \\
\text {. }\end{array}$ & $\begin{array}{l}\hat{\infty} \\
\overrightarrow{+} \\
\dot{q}\end{array}$ & $\begin{array}{l}\stackrel{N}{f} \\
\stackrel{\sigma}{0}\end{array}$ & $\begin{array}{l}\infty \\
\infty \\
\infty \\
0\end{array}$ & $\begin{array}{l}\stackrel{2}{\infty} \\
\infty\end{array}$ & 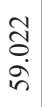 & $\begin{array}{l}\mathbb{b} \\
\stackrel{n}{n} \\
\stackrel{n}{-}\end{array}$ & $\begin{array}{l}a \\
\vec{b} \\
\dot{ \pm}\end{array}$ \\
\hline & 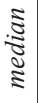 & $\stackrel{\leftrightarrow}{\stackrel{f}{*}}$ & 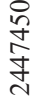 & $\begin{array}{l}\bar{m} \\
\dot{q}\end{array}$ & $\begin{array}{l}\stackrel{0}{ } \\
\infty \\
\dot{J} \\
\dot{J}\end{array}$ & $\begin{array}{l}\forall \\
\stackrel{J}{n} \\
m\end{array}$ & तె & $\begin{array}{l}\frac{N}{\sigma} \\
0 \\
0\end{array}$ & $\overrightarrow{\widehat{a}}$ & f. & $\underset{\infty}{\sigma}$ & $\begin{array}{l}\vec{I} \\
\dot{I}\end{array}$ \\
\hline & 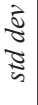 & $\hat{\sigma}$ & \begin{tabular}{l} 
O \\
+ \\
\multirow{2}{*}{} \\
i
\end{tabular} & $\begin{array}{l}\stackrel{\infty}{+} \\
\stackrel{d}{\sim}\end{array}$ & $\begin{array}{l}m \\
m \\
\sim \\
\sim\end{array}$ & $\begin{array}{l}m \\
\exists \\
\stackrel{m}{m}\end{array}$ & $\begin{array}{l}m \\
\sigma \\
\infty \\
\sim\end{array}$ & $\stackrel{g}{\sigma}$ & $\begin{array}{l}\hat{S} \\
\text { + } \\
\text { I }\end{array}$ & $\begin{array}{l}\stackrel{\infty}{\frac{\pi}{m}} \\
\frac{1}{1}\end{array}$ & 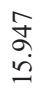 & $\begin{array}{l}n \\
\varrho \\
i \\
i\end{array}$ \\
\hline & $\Xi$ & $\stackrel{0}{0}$ & $\begin{array}{l}\infty \\
0 \\
0 \\
\text { N } \\
\stackrel{n}{n}\end{array}$ & $\begin{array}{l}\hat{\sigma} \\
\dot{\infty}\end{array}$ & 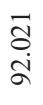 & $\begin{array}{l}0 \\
\stackrel{1}{8} \\
0\end{array}$ & $\stackrel{\square}{\stackrel{D}{\infty}}$ & 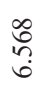 & 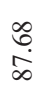 & $\frac{n}{a}$ & $\begin{array}{l}\vec{\sim} \\
\tilde{n} \\
\infty \\
\infty\end{array}$ & $\begin{array}{l}\infty \\
\infty \\
\infty \\
\infty \\
\infty\end{array}$ \\
\hline & $\stackrel{\stackrel{\Xi}{\Xi}}{\Xi}$ & $\dot{\varphi}$ & $\begin{array}{l}8 \\
\text { రై } \\
\end{array}$ & $\stackrel{\infty}{=}$ & 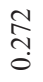 & $\stackrel{n}{\dot{m}}$ & $\begin{array}{l}\infty \\
\infty \\
\stackrel{+}{\beth}\end{array}$ & F⿱ & $\vec{\sigma}$ & $\begin{array}{l}\infty \\
\\
\sim\end{array}$ & 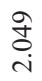 & $\begin{array}{l}\infty \\
\hat{\sigma} \\
\stackrel{a}{ }\end{array}$ \\
\hline & 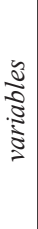 & ్ָర & 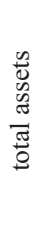 & 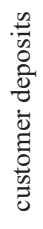 & 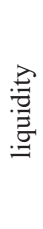 & 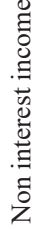 & 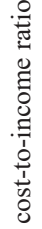 & 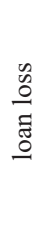 & 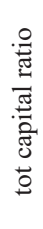 & $\begin{array}{l}\mathscr{\Xi} \\
\stackrel{\Xi}{\Xi} \\
\stackrel{\Xi}{\Xi}\end{array}$ & 坣 & $\begin{array}{l}0 \\
0 \\
0 \\
\tilde{\Xi} \\
\Xi \\
\Xi\end{array}$ \\
\hline
\end{tabular}

\begin{tabular}{|c|c|c|c|c|c|c|c|c|c|c|c|c|}
\hline & $\begin{array}{l}\vec{े} \\
0 \\
\vdots \\
\vdots\end{array}$ & $\underset{⿱ 亠}{\Delta}$ & $\underset{\frac{N}{N}}{\stackrel{N}{N}}$ & 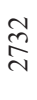 & $\frac{\mathfrak{Y}}{\stackrel{N}{N}}$ & $\stackrel{\text { సે}}{\curvearrowright}$ & $\stackrel{\infty}{\curvearrowright}$ & $\frac{0}{\sqrt{N}}$ & $\begin{array}{l}\infty \\
\hat{\overbrace{}} \\
\sim\end{array}$ & 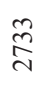 & $\stackrel{\stackrel{N}{\sim}}{\sim}$ & $\frac{\stackrel{+}{ \pm}}{\Delta}$ \\
\hline 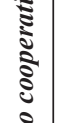 & $\begin{array}{l}\Xi \\
\Xi\end{array}$ & $\begin{array}{l}0 \\
\stackrel{0}{0} \\
0\end{array}$ & $\underset{\frac{9}{i}}{\stackrel{9}{*}}$ & $\frac{a}{\hat{b}}$ & $\frac{2}{d}$ & $\begin{array}{l}\hat{\delta} \\
\text {. } \\
\text { i }\end{array}$ & $\begin{array}{l}n \\
\tilde{n} \\
\tilde{o} \\
0\end{array}$ & $\begin{array}{l}\infty \\
\infty \\
\infty \\
0\end{array}$ & $\begin{array}{l}\frac{n}{a} \\
\end{array}$ & $\begin{array}{l}\vec{J} \\
\text { in } \\
0\end{array}$ & $\stackrel{\Omega}{\Omega}$ & $\begin{array}{l}\stackrel{n}{n} \\
\stackrel{\sim}{n}\end{array}$ \\
\hline 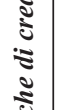 & 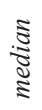 & $\begin{array}{l}0 \\
\stackrel{n}{0}\end{array}$ & 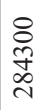 & $\begin{array}{l}\frac{+}{\infty} \\
\dot{\sigma} \\
\dot{\sigma}\end{array}$ & $\begin{array}{l} \pm \\
n \\
\infty\end{array}$ & $\begin{array}{l}\overline{\widehat{a}} \\
\text { ה }\end{array}$ & $\begin{array}{l}\hat{\sigma} \\
\dot{8}\end{array}$ & $\begin{array}{l}\infty \\
\text { ñ. } \\
0\end{array}$ & $\begin{array}{l}n \\
\sigma \\
\ddot{0} \\
-\end{array}$ & 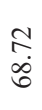 & $\begin{array}{l}\hat{b} \\
\infty \\
0 \\
0\end{array}$ & 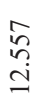 \\
\hline $\begin{array}{l}0 \\
5 \\
\Xi \\
\Xi\end{array}$ & 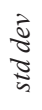 & 胥 & 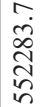 & $\begin{array}{l}\text { J } \\
\text { i }\end{array}$ & $\begin{array}{l}n \\
=\end{array}$ & $\stackrel{\bar{\sigma}}{\circ}$ & $\begin{array}{l}\hat{2} \\
\hat{\sigma} \\
\dot{n}\end{array}$ & $\begin{array}{l}0 \\
\infty \\
0 \\
0 \\
0\end{array}$ & $\begin{array}{l}\vec{\partial} \\
\dot{\infty}\end{array}$ & $\begin{array}{l}\stackrel{0}{f} \\
\stackrel{r}{-}\end{array}$ & $\begin{array}{l}8 \\
\infty \\
0 \\
0\end{array}$ & $\stackrel{\circ}{\circ}$ \\
\hline 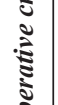 & $\stackrel{\Xi}{\leftrightarrows}$ & $\stackrel{n}{0}$ & 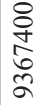 & $\begin{array}{l}\tilde{2} \\
\text { } \\
\dot{\infty}\end{array}$ & $\begin{array}{l}\vec{\sigma} \\
\text { d } \\
\text { }\end{array}$ & $\begin{array}{l}\text { ?े } \\
\text { aे }\end{array}$ & 至 & $\begin{array}{l}\infty \\
0 \\
n \\
0 \\
0\end{array}$ & $\underset{\infty}{\infty}$ & $\begin{array}{l}\vec{b} \\
\infty \\
\infty\end{array}$ & $\begin{array}{l}\vec{\sim} \\
\tilde{n} \\
\infty\end{array}$ & $\begin{array}{l}\tilde{2} \\
0 \\
0\end{array}$ \\
\hline & $\stackrel{\Xi}{\Xi}$ & $\hat{\varphi}$ & $\begin{array}{l}8 \\
\text { రુ } \\
\text { - }\end{array}$ & $\begin{array}{l}0 \\
\infty \\
\text { ஸे }\end{array}$ & $\frac{N}{\pi}$ & $\frac{\bar{a}}{\bar{r}}$ & $\begin{array}{l}0 \\
\stackrel{0}{0} \\
\stackrel{1}{0}\end{array}$ & $\begin{array}{l}n \\
\mathfrak{o} \\
0 \\
0 \\
1\end{array}$ & $\hat{\sigma}$ & $\stackrel{ \pm}{I}$ & 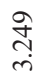 & $\begin{array}{l}\infty \\
\stackrel{\infty}{\sigma} \\
\stackrel{a}{ }\end{array}$ \\
\hline \multirow{7}{*}{ 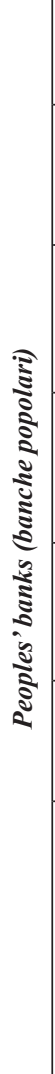 } & $\begin{array}{l}\overline{5} \\
\overrightarrow{0} \\
\vdots\end{array}$ & $\stackrel{\text { F }}{m}$ & $\stackrel{\mathfrak{Y}}{\mathrm{m}}$ & $\stackrel{\mathfrak{Y}}{m}$ & $\stackrel{\mathfrak{F}}{\mathrm{f}}$ & $\stackrel{\text { I }}{m}$ & $\stackrel{\mathfrak{T}}{\mathrm{f}}$ & ले & లి & $\stackrel{\text { I }}{m}$ & $\stackrel{\mathscr{Y}}{\mathrm{m}}$ & $\stackrel{\text { Fे }}{m}$ \\
\hline & $\begin{array}{l}\tilde{\Xi} \\
\Xi\end{array}$ & $\stackrel{\substack{f \\
0}}{0}$ & \begin{tabular}{l}
$\hat{0}$ \\
0 \\
0 \\
\multirow{j}{0}{} \\
\end{tabular} & 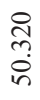 & $\begin{array}{l}\hat{a} \\
\stackrel{2}{2}\end{array}$ & $\begin{array}{l}8 \\
\text { i } \\
\text { n' }\end{array}$ & $\begin{array}{l}n \\
0 \\
0 \\
\dot{b}\end{array}$ & 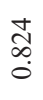 & $\begin{array}{l}\stackrel{N}{a} \\
\dot{J}\end{array}$ & $\begin{array}{l}\infty \\
\stackrel{0}{0} \\
\stackrel{2}{2} \\
0\end{array}$ & 웅 & $\begin{array}{l}\hat{6} \\
\stackrel{n}{n}\end{array}$ \\
\hline & 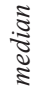 & 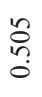 & 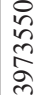 & $\begin{array}{l}\hat{\infty} \\
\stackrel{1}{n} \\
\stackrel{n}{n}\end{array}$ & 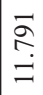 & $\frac{\sim}{\sim}$ & $\begin{array}{l}n \\
q \\
\dot{b}\end{array}$ & $\begin{array}{l}0 \\
\stackrel{0}{0}\end{array}$ & $\begin{array}{l}\infty \\
\stackrel{\infty}{0} \\
\stackrel{\sim}{-}\end{array}$ & $\stackrel{\grave{n}}{\tilde{r}}$ & $\begin{array}{l}\dot{\partial} \\
\infty \\
\sigma\end{array}$ & $\begin{array}{l}\stackrel{n}{a} \\
\stackrel{n}{n}\end{array}$ \\
\hline & $\frac{\vec{a}}{\tilde{z}}$ & $\begin{array}{l}n \\
\infty \\
n \\
0\end{array}$ & 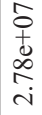 & 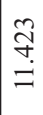 & $\begin{array}{l}\mathfrak{g} \\
\hat{a} \\
\text { in }\end{array}$ & $\begin{array}{l}\stackrel{g}{d} \\
\stackrel{1}{N}\end{array}$ & $\begin{array}{l}\bar{\delta} \\
\stackrel{1}{c}\end{array}$ & $\begin{array}{l}\text { तु } \\
\text { லீ }\end{array}$ & 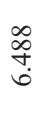 & $\begin{array}{l}\stackrel{n}{g} \\
\dot{m}\end{array}$ & 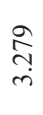 & 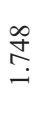 \\
\hline & $\stackrel{\Xi}{\leftrightarrows}$ & $\begin{array}{l}\stackrel{0}{i} \\
i\end{array}$ & 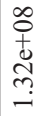 & $\begin{array}{l}\infty \\
\infty \\
\infty \\
\infty \\
\infty\end{array}$ & $\begin{array}{l}\tilde{y} \\
\dot{n} \\
\infty\end{array}$ & $\begin{array}{l}\infty \\
\infty \\
\dot{\sigma}\end{array}$ & $\begin{array}{l}0 \\
\stackrel{2}{1}\end{array}$ & $\begin{array}{l}n \\
\stackrel{n}{n} \\
+\end{array}$ & $\begin{array}{l}\stackrel{+}{+} \\
\stackrel{+}{n}\end{array}$ & ț. & $\begin{array}{l}\stackrel{o}{\vec{v}} \\
\dot{d} \\
\stackrel{d}{d}\end{array}$ & $\begin{array}{l}\vec{\rho} \\
\infty \\
\infty\end{array}$ \\
\hline & ミ & $\underset{\stackrel{\jmath}{i}}{\stackrel{r}{i}}$ & 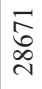 & 泾 & $\frac{n}{\hat{\sigma}}$ & $\bar{\sigma}$ & $\frac{\pi}{i}$ & $\begin{array}{l}\vec{J} \\
\stackrel{0}{0}\end{array}$ & $\begin{array}{l}\vec{\gamma} \\
\dot{0}\end{array}$ & $\frac{\infty}{\stackrel{+}{+}}$ & $\begin{array}{l}\tilde{b} \\
\tilde{r} \\
\dot{\gamma}\end{array}$ & $\begin{array}{l}\text { ro } \\
\text { ִִ } \\
0\end{array}$ \\
\hline & 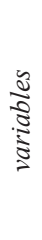 & ఝ్ర & 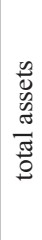 & 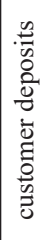 & 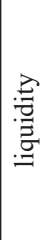 & 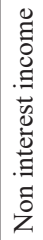 & 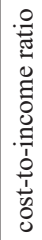 & 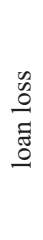 & 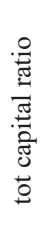 & $\begin{array}{l}\mathscr{U} \\
\stackrel{\Xi}{\Xi} \\
\frac{\Xi}{\Xi}\end{array}$ & 坣 & $\begin{array}{l}\frac{n}{0} \\
0 \\
a \\
\Xi \\
\Xi\end{array}$ \\
\hline
\end{tabular}


and heteroscedasticity of the error term ${ }^{7}$.

Heteroscedasticity issues and serial correlation are dealt with by clustering standard errors at the bank (bank type) level. To address multicollinearity concerns, the correlation coefficients are calculated and reported in Table 2 below. As indicated in the table, none of the coefficients are very high (none are above 0.50 for example, except net loans on assets and non interest income on revenues), which reduces possible concerns over multicollinearity. In addition, the variance inflation factors (VIFs; see Table 3 below) have been calculated to assess whether there is multicollinearity within the data $^{8}$. The values are within the normal range (under 2) and thus indicate that there should be no major concerns over multicollinearity.

The strongest correlations are between liquid assets on customer \& short term funding and net loans on assets $(-0.72)$, between non interest income on revenues and equity on assets $(0.45)$ and between non interest income on revenues and liquid assets on customer \& short term funding (0.40).

Since VIF value are all below 2, there appears to be no multicollinearity in the data. The only value close to 2 is that of liquid assets on customer short term funding, nonetheless, being lower than 2, it appears to be acceptable.

1.1.1 Regression results

The main results of the empirical analysis are presented

Table 2: Correlation coefficients for the main explanatory variables (Source: Author's calculations)

\begin{tabular}{|c|c|c|c|c|c|c|}
\hline \multirow[b]{2}{*}{ variables } & \multicolumn{6}{|c|}{ correlation matrix } \\
\hline & {$[1]$} & {$[2]$} & [3] & [4] & [5] & [6] \\
\hline [1] log total assets & 1 & & & & & \\
\hline [2] customer deposits on assets & -0.348302 & 1 & & & & \\
\hline [3] liquid assets on customer s.t. funding & -0.054354 & 0.03378 & 1 & & & \\
\hline [4] non interest income on revenues & 0.1646632 & -0.05749 & 0.4006 & 1 & & \\
\hline [5] net loans on assets & 0.2214717 & -0.24772 & -0.7245 & -0.35991 & 1 & \\
\hline [6] equity on assets & -0.352166 & -0.0234 & 0.4581 & 0.179078 & -0.39339 & 1 \\
\hline
\end{tabular}

Table 3: VIF estimates (Source: Author's calculations)

\begin{tabular}{|c|c|}
\hline \multicolumn{2}{|c|}{ Variance inflation factors } \\
\hline variables & VIF value \\
\hline log total assets & 1.476995791 \\
\hline customer deposits on assets & 1.320341176 \\
\hline liquid assets on customer \& short-term funding & 1.997004493 \\
\hline non interest income on revenues & 1.08075627 \\
\hline net loans on assets & 1.703055281 \\
\hline equity on assets & 1.710629854 \\
\hline cooperative banks & 1.303943469 \\
\hline crisis year & 1.020241787 \\
\hline savings banks & 1.484342875 \\
\hline popular banks & 1.501541947 \\
\hline
\end{tabular}

\footnotetext{
${ }^{7}$ Unfortunately, the time-invariant nature of the explanatory variable prevents one from using panel data estimators that would allow to better control for reverse causality and, most importantly, the unobserved heterogeneity problem (i.e. endogeneity). In fact, a superior solution would be to rely on the fixed effects linear estimator, i.e. to control for the firm fixed effects in the regressions or, alternatively, to use a dynamic linear panel estimator, which would also account for the dynamic endogeneity of some of our variables (Wintoki et al., 2012). However, while addressing the endogeneity of some of the variables, using firm fixed effects would prevent one from estimating the coefficients for all the time-invariant variables, such as the main variable of interest, the bank type. ${ }^{8}$ The VIF for a covariate is the reciprocal of 1 minus the multiple R-squared for the regression of the covariate on the other covariates. If the $j$ th covariate is orthogonal to the other covariates, the multiple R-squared of the regression of the $j$ th covariate on the other covariates will be equal to 0 and therefore the VIF will be equal to 1 . On the other hand, if there is multicollinearity, the R-squared will be close to 1 and therefore the VIF will be high. In the case of the dummy variable representing bank type, McFadden's pseudo R-squared is used to calculate the VIF.
} 
in Tables $4-6$ below. To start with, the basic model is discussed, which to some extent follows Ferri et al. (2010). Unlike their sample, the sample in the present study covers only Italian banks and includes the period of the financial crisis, which is defined as the years 2009-2012 (or alternatively as 2008-2012; see robustness section). In model (1), a pooled OLS is estimated using the ROAA, COST_EFFICIENCY and LOAN_PROVISIONS as the dependent variables with the standard errors clustered at the individual bank level. The main explanatory variables are dummies for the different bank types (the commercial banks being the reference group). Model (2) replicates model (1) with the exception that here the standard errors are clustered by bank type. Model (3) replicates model (1) and additionally includes an interaction term (COOP*CRISIS) that aims to capture differences in the eventual effects of the crisis for cooperative banks in comparison to other banks.

Models (4)- (7) are estimated using the fixed effects linear estimator, which means that one controls for any time-invariant unobserved bank characteristics. Due to this, one cannot estimate the coefficient of the main explanatory variable, i.e. the dummy for cooperative banks. Following Lins et al.'s (2013) study on family firms' performance during the crisis, the focus is therefore on the coefficient of the interaction term COOP*CRISIS, which should measure the differences between the cooperative banks' and other banks' crisis performance. Models (4) and (5) differ in relation to the clustering of standard errors (i.e. more conservative clustering by bank type in model (4), and clustering at the bank level in model (5)). Models (6) and (7) replicate models (4) and (5) but here the people's banks are excluded from the sample, with the purpose of comparing cooperative banks with profit-oriented banks only.

With regards to the return on the banks' average assets (ROAA), a higher profitability is observed for the banks with a higher share of non-interest income and for banks with a higher share of equity. Moreover, cooperative banks on average associate with slightly higher return on assets than other banks ( 28 basis points over the entire period of analysis; see the positive coefficient for COOP in model (1) and model (2)), which is compatible with Hypothesis 1. Other types of banks do not seem to perform any different than commercial banks, which are the reference group of banks in the analysis. Here one must note that-despite controlling for a set of bank-specific characteristics, such as the share of equity and the share of non-interest income - the positive coefficient for cooperative banks should be interpreted with caution. In fact, it is quite likely that this coefficient still captures some unobserved characteristic that are more common in cooperative banks and that also influence bank profitability. Moreover, the superior performance of cooperative banks during the entire period may be primarily due to the (expected) better performance of these banks during the crisis. To account for this, in model (3) an interaction term COOP*CRISIS is introduced and, furthermore, a dummy for the entire crisis period (i.e. after 2009) included at the place of corresponding time dummies. As indicated in the table, the crisis period associates with a significant drop in banks' performance, i.e. the coefficient for the dummy CRISIS is negative and statistically significant, and thus confirms Hypothesis 2. Moreover, the positive coefficient for the interaction term COOP*CRISIS suggests that the effects of the crisis were lower for cooperative banks in comparison to other banks. While this is in line with the Hypothesis 3, the coefficient is not statistically significant across the various specifications (i.e. see model (3) - model (7) in Table 4).

Next, banks' efficiency is accounted for and measured by the cost-to-income ratio (COST_EFFICIENCY) in Table 5 below. The specifications of the various models follow the analysis of ROAA (see above). First, larger banks associate with higher efficiency, i.e. lower costs. The coefficient for total bank assets is negative and statistically significant also when one includes firm fixed effects in models (4)-(7). This result is in line with the expectations, as larger banks are more likely to realize economies of scale, which implies lower costs. On the other hand, a positive relationship between the share of customer deposits and bank costs is observed. This again makes sense as one could expect that the banks, which rely more on depositors as a source of financing, also have a higher number of branches and employees (and consequently, higher operation costs) in order to gain better access to current and new depositors.

As shown in Table 5, cooperative banks associate with lower operating costs (everything else equal, the cost-toincome ratio is about 4.7-4.8 percentage points lower), which is in line with Hypothesis 1 above. Other banks also incurred a significant increase in their costs during the crisis period, i.e. by about 8.7 percentage points on average (see the coefficient for the CRISIS dummy in the fixed effects specification, for example). As for the effect of the crisis on cooperative credit banks specifically, the interaction term between the two dummies, one indicating cooperative credit banks and the other crisis years, is positive in fixed effect models and negative in model (3), but in all cases not significantly different from 0 , so it does not fully contradict Hypothesis 3.

A better picture for cooperative credit banks emerges when looking at their loan quality during the entire period and the crisis (LOAN_PROVISIONS). As reported in Table 6, cooperative banks associate with better loan quality, which is captured in this case by the share of loan loss provisions in the value of gross bank loans $(0,16-$ 0,19 percentage points lower for cooperative credit banks compared to commercial banks). The other banks' loan quality deteriorated significantly during the crisis period, 
Table 4: ROAA and bank organizational form (Source: Author's calculations)

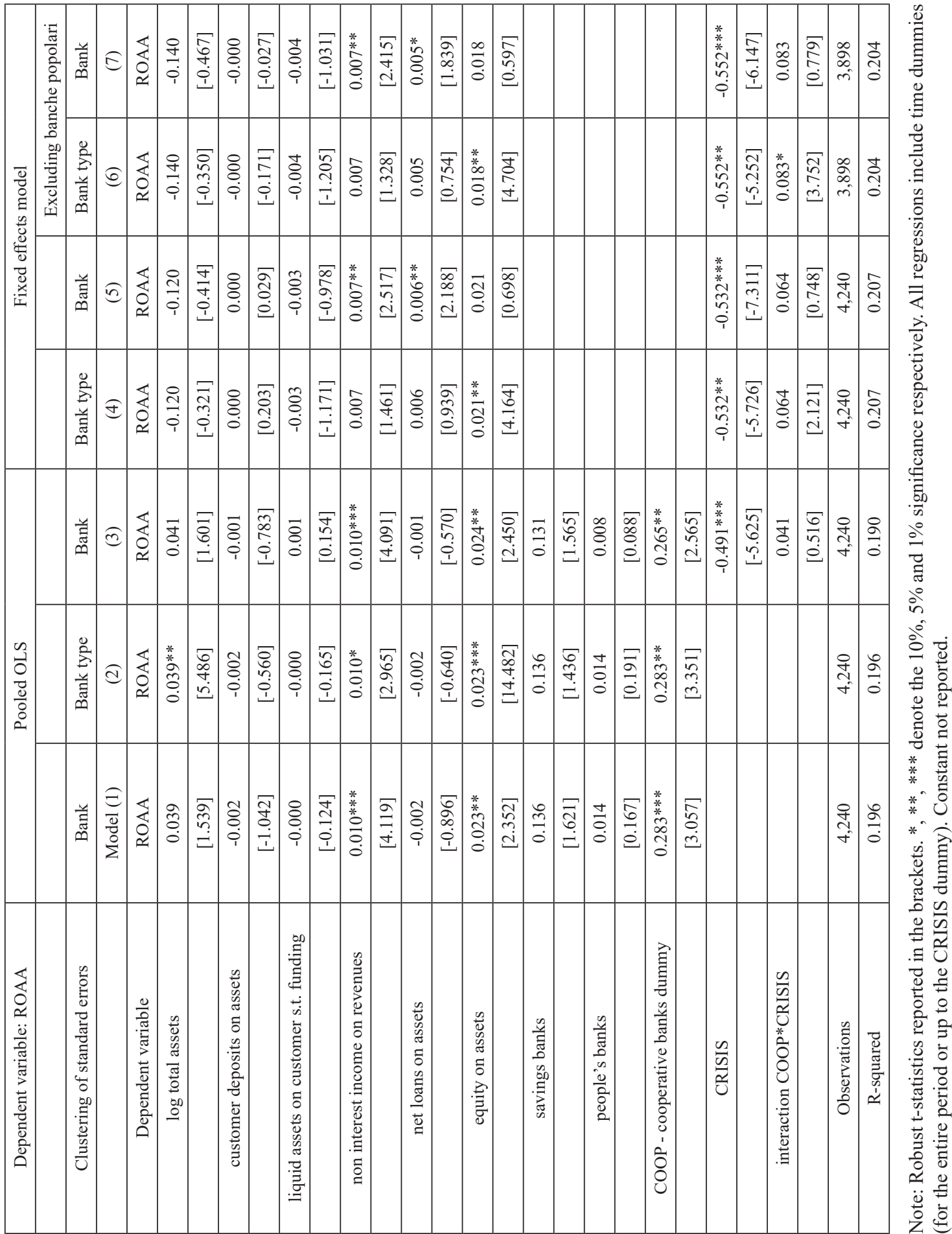


Table 5: Cost-income ratio (COST_EFFICIENCY) and bank organizational form (Source: Author's calculations)

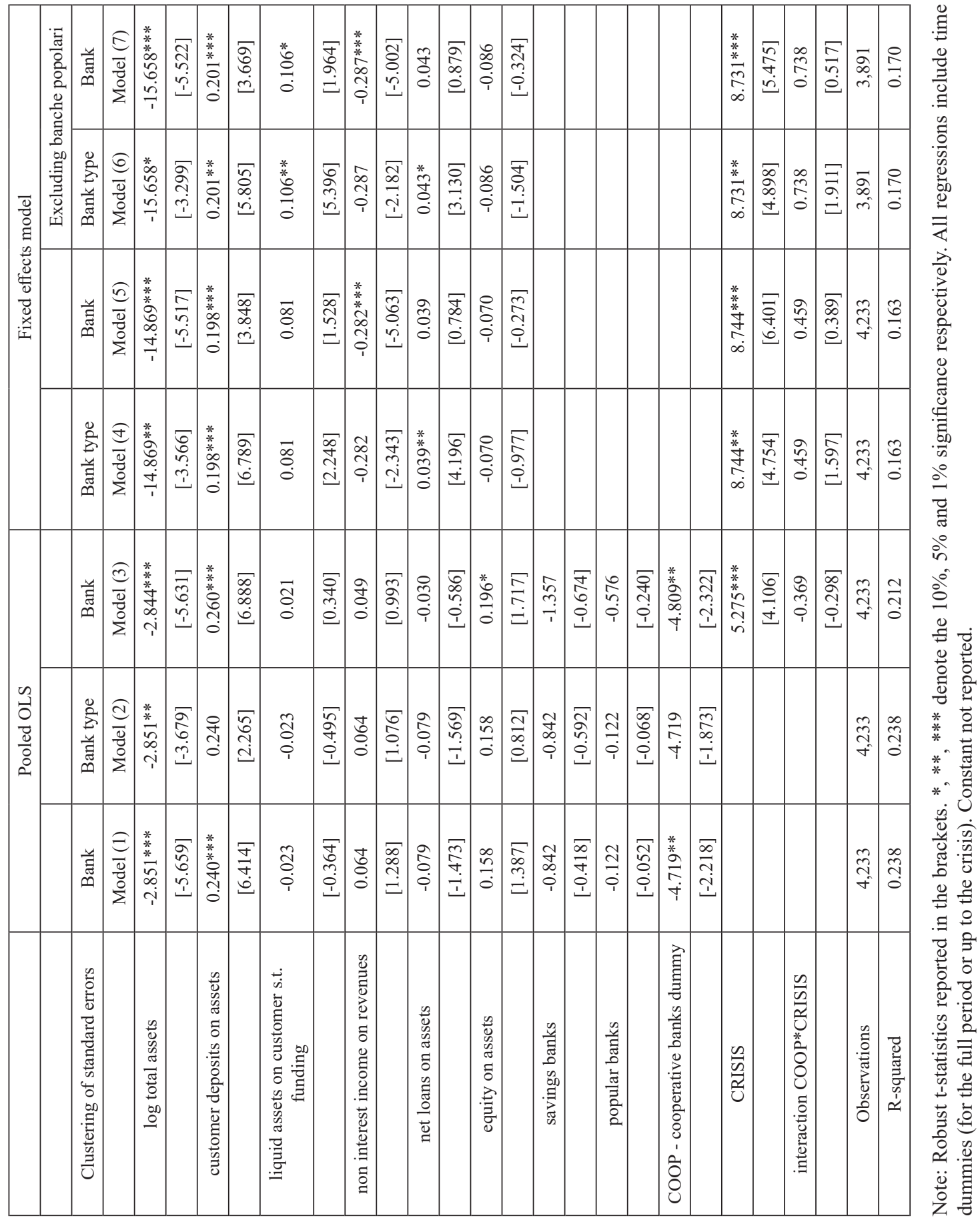


with loan loss provisions increasing 0.26 to 0.53 percentage points of gross loans. On the other hand, the cooperative credit banks associate with a lower increase in the loan loss provisions during the period of the crisis, which suggests that the quality of their loans did not deteriorate as much as for other banks (see the positive and statistically significant coefficient for the interaction term COOP*CRISIS in the fixed effects models). These results provide support to the hypotheses stated above (Hypothesis 1-3). Moreover, it seems that the loan quality overall positively associates with the soundness of bank operations and, somehow, with more traditional types of bank activities; loan loss provisions are smaller for the banks with a higher share of equity, stronger bank liquidity, a higher share of customer deposits and a smaller share of non-interest income (see the fixed effects results in model (4)-model (7)).

\section{Discussion and conclusion}

Through a comparative study on profitability, efficiency and lending policies between different types of banks in Italy, this paper shows that Italian cooperative credit banks indeed operate differently than standard commercial banks. The paper has tested the hypothesis that cooperative banks offset their lower profitability with a more efficient asset allocation policy. Specifically, the main hypothesis that has been investigated is whether Italian cooperative banks have more conservative policies and lower riskiness of bank operations than commercial banks. The argument that such banks rely on a specific model of banking is thus confirmed by the present analysis. Stated otherwise, the model developed in this paper shows the importance of taking bank typologies into account when discussing bank's behaviour both in normal times and during periods of distress.

The paper shows that different banking models have reacted differently to the financial crisis and economic downturn. This has important implications for regulation policies in the aftermath of the crisis. The model developed in this paper stresses differences between bank types, an issue that has been often neglected by the mainstream literature in the past. Present results seem to confirm the validity of recent proposals developed by the Italian Government to treat different banks differently with the aim to improve their governance mechanisms, features and institutional characteristics (Stefancic, 2015; Il Sole 24 Ore, 2015; The Economist, 2015; Ferraresi et al., 2016). Everything else equal, cooperative banks in Italy seem to be relatively more efficient than commercial counterparts. Arguably, they tend to enjoy a good market positioning. On the one hand, the above results appear to be aligned with that of Ferri et al. (2010); on the other hand, they tend to depart from the argument stating that mutual and cooperative banks are less efficient than commercial banks (see e.g. Rasmusen 1988). While acknowledging that profitability has decreased in all types of banks as a result of the credit crisis and the economic downturn, the main findings of the paper can be summarised as follows:

- Cooperative credit banks tend to have a more efficient asset allocation policy, as shown by a higher ROAA.

- As to cost-to-income ratios, larger banks show economies of scale, yet cooperative banks show, in some models, a significantly lower cost-to-income ratio (around 4.8 percentage points lower) than comparable commercial banks. This result would suggest that cooperative banks are organizationally more efficient, although further research on the topic is needed before drawing definitive conclusions.

- Customer deposits appear to have an effect on the cost-to-income ratio. This result suggests a critical reassessment of cooperative banks' branch network policy, the optimal density of the network of branches and related (unforeseen) costs.

- With regards to the quality of credit, the crisis has significantly impacted on loan loss provisions as a share of gross loans, with a marked increase for all banks. The deterioration of credit has been, however, less severe for cooperative credit banks, which confirms their more prudent lending policies.

Needless to say, these findings should be of relevance to managers in Italian banks. One should acknowledge the fact that the results are valid for the Italian banking market only, and are limited to the period 2005-2012. Additional research focusing on banks from other European countries is needed in order to capture subsequent developments and extend the suggested policies to the EU level. Nonetheless, substantial business implications can be derived and applied to those EU countries where the cooperative banking sector is not yet fully developed (as for instance in former Yugoslavian countries or some countries in Eastern Europe). Furthermore, based on the results of the present paper, the following suggestions in terms of market regulation can be made: 
Table 6: Loan loss provisions (LOAN_PROVISIONS) and bank organizational form (Source: Author's calculations)

\begin{tabular}{|c|c|c|c|c|c|c|c|c|c|c|c|c|c|c|c|c|c|c|c|c|c|c|c|c|c|c|}
\hline \multirow{4}{*}{ 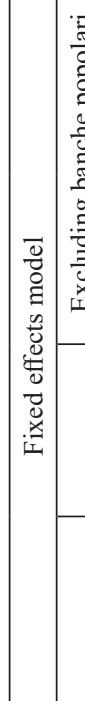 } & 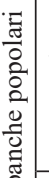 & $\begin{array}{l}\text { 范 } \\
\text { | }\end{array}$ & $E$ & $\begin{array}{l}\hat{\tilde{a}} \\
\dot{0} \\
\dot{1}\end{array}$ & 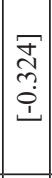 & 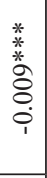 & 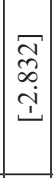 & $\mid \begin{array}{l}* \\
* \\
* \\
o \\
o \\
o \\
\dot{0} \\
i\end{array}$ & 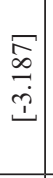 & | & 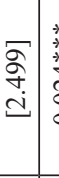 & & 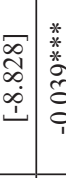 & 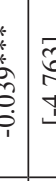 & 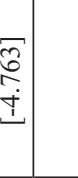 & & & & & & 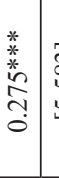 & 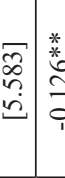 & 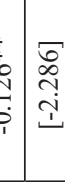 & & 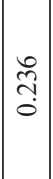 & $\begin{array}{l}\stackrel{0}{E} \\
\stackrel{0}{0} \\
\stackrel{0}{0}\end{array}$ \\
\hline & 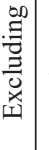 & 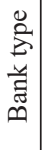 & $\theta$ & $\begin{array}{l}\hat{\hat{~}} \\
\dot{i} \\
\dot{i}\end{array}$ & $\left|\begin{array}{c}2 \\
0 \\
0 \\
\dot{1} \\
\dot{1}\end{array}\right|$ & $\mid \begin{array}{l}0 \\
\vdots \\
0 \\
\dot{1}\end{array}$ & 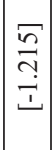 & $\mid \begin{array}{l}\infty \\
0 \\
0 \\
\dot{1} \\
1\end{array}$ & 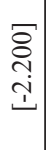 & $\begin{array}{l}\swarrow \\
\vdots \\
0\end{array}$ & $\begin{array}{c}\overline{\widehat{C}} \\
\stackrel{\Xi}{\Xi}\end{array}$ & 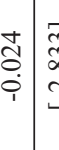 & 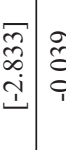 & 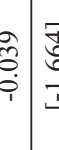 & 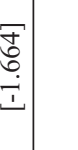 & & & & & & 葵 & 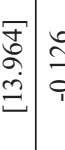 & 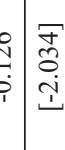 & & 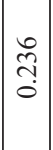 & \\
\hline & & 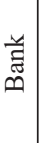 & $\approx$ & $\begin{array}{l}9 \\
0 \\
0 \\
i\end{array}$ & \begin{tabular}{|c|} 
\\
0 \\
$\dot{+}$ \\
$\dot{1}$ \\
$\dot{1}$ \\
\end{tabular} & 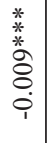 & 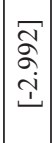 & 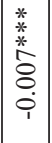 & 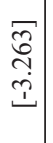 & $\begin{array}{l}* \\
* \\
* \\
* \\
* \\
\vdots \\
\vdots \\
0\end{array}$ & 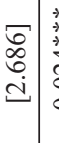 & 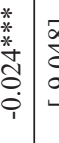 & 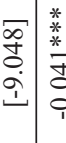 & 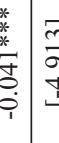 & 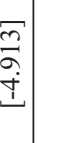 & & & & & & . & 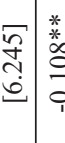 & 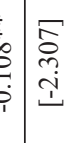 & & ָे. & 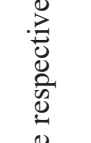 \\
\hline & & 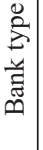 & 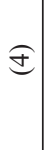 & $\begin{array}{l}0 \\
0 \\
0 \\
i\end{array}$ & 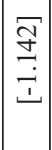 & 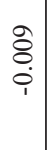 & 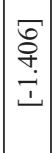 & 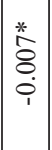 & $\begin{array}{l}\overrightarrow{\vec{\infty}} \\
\stackrel{+}{+} \\
\stackrel{1}{i}\end{array}$ & $\begin{array}{l}n \\
\vdots \\
\vdots \\
\circ\end{array}$ & 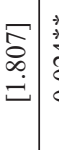 & 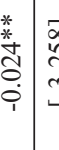 & 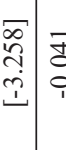 & 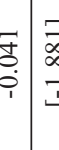 & \begin{tabular}{|l|}
$\overrightarrow{\vec{D}}$ \\
$\infty$ \\
$\dot{\square}$ \\
\end{tabular} & & & & & & . & 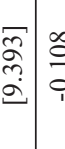 & 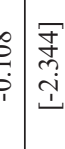 & & స્స్ & $\begin{array}{l}.07 \\
.50 \\
0 \\
0 \\
0\end{array}$ \\
\hline \multirow{3}{*}{ 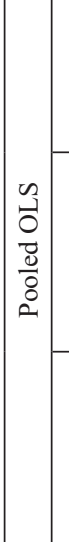 } & & चै & (્) & 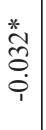 & 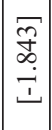 & 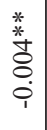 & 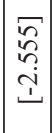 & 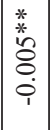 & 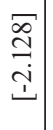 & $\begin{array}{l}\overrightarrow{8} \\
\dot{0} \\
\dot{1}\end{array}$ & 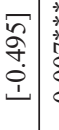 & 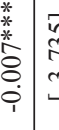 & 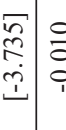 & 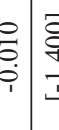 & 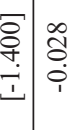 & $\begin{array}{c}\bar{p} \\
\tilde{f} \\
0 \\
\dot{1} \\
\end{array}$ & \begin{tabular}{|l|}
$\infty$ \\
0 \\
0 \\
0 \\
0
\end{tabular} & $\begin{array}{l}\bar{\curvearrowright} \\
\tilde{\sigma} \\
\dot{e}\end{array}$ & $\mid$\begin{tabular}{|}
$*$ \\
$*$ \\
0 \\
0 \\
0 \\
\\
1 \\
1
\end{tabular} & 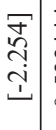 & | & 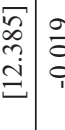 & 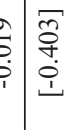 & \begin{tabular}{l}
$\stackrel{+}{n}$ \\
\multirow{f}{*}{}
\end{tabular} & 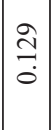 & $\mathrm{g}^{\circ}$ \\
\hline & & 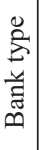 & $\widehat{\mathbb{d}}$ & $\begin{array}{l}0 \\
0 \\
0 \\
i \\
i\end{array}$ & 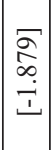 & 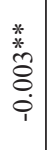 & $\begin{array}{l}\widetilde{T} \\
\stackrel{T}{\dot{J}} \\
\dot{J}\end{array}$ & $\begin{array}{l}\overrightarrow{8} \\
\dot{0} \\
\dot{i} \\
\end{array}$ & 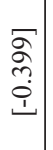 & 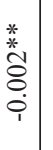 & \begin{tabular}{c|c}
$\overline{\vec{\tau}}$ \\
$\vec{\sim}$ \\
$\dot{\vec{t}}$
\end{tabular} & 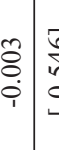 & 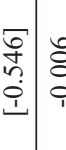 & 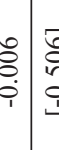 & 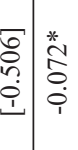 & 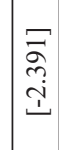 & $\begin{array}{l}0 \\
0 \\
0 \\
0 \\
0\end{array}$ & $\begin{array}{l}\overline{\bar{\lambda}} \\
\stackrel{\Xi}{\Xi}\end{array}$ & 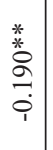 & $\begin{array}{l}\infty \\
\stackrel{\infty}{f} \\
\dot{\varphi} \\
\end{array}$ & & & & \begin{tabular}{l}
$\stackrel{7}{0}$ \\
\multirow{*}{*}{}
\end{tabular} & $\begin{array}{l}n \\
\\
0\end{array}$ & $\begin{array}{l}\vec{E} \\
\text { E } \\
*\end{array}$ \\
\hline & & है & 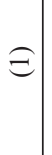 & $\begin{array}{l}0 \\
0 \\
0 \\
0 \\
0 \\
0 \\
10\end{array}$ & 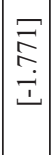 & $\mid \begin{array}{l}0 \\
\hat{0} \\
\dot{i}\end{array}$ & $\mid \begin{array}{c}n \\
0 \\
n \\
\vdots \\
ت \\
\end{array}$ & $\begin{array}{l}\overrightarrow{8} \\
\dot{8} \\
\dot{\varphi}\end{array}$ & $\begin{array}{l}\sigma \\
\vec{\lambda} \\
\hat{i} \\
\dot{1}\end{array}$ & 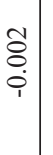 & 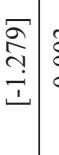 & 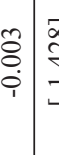 & 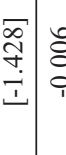 & 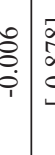 & 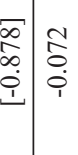 & $\mid \begin{array}{l}\bar{D} \\
\infty \\
\infty \\
\dot{1} \\
\dot{1}\end{array}$ & $\mid \begin{array}{l}0 \\
0 \\
0 \\
0 \\
0\end{array}$ & $\begin{array}{c}\sigma \\
\tilde{f} \\
\dot{e}\end{array}$ & 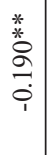 & 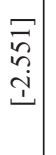 & & & & 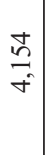 & $\begin{array}{l}\stackrel{n}{\hat{T}} \\
0\end{array}$ & 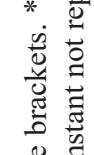 \\
\hline & & 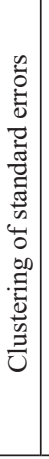 & & 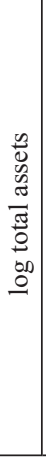 & & 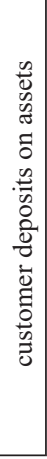 & & 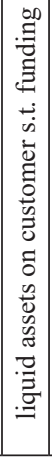 & & 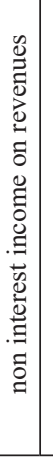 & & 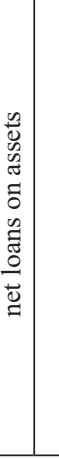 & & 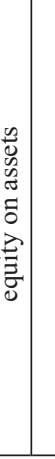 & 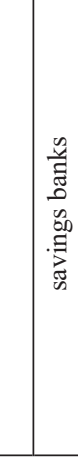 & & 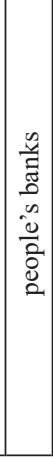 & & 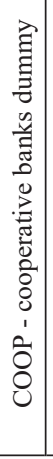 & & 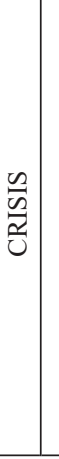 & 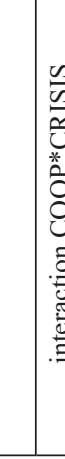 & & & 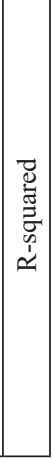 & 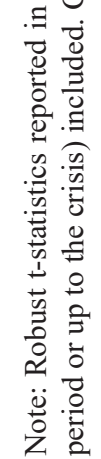 \\
\hline
\end{tabular}


- Having assessed the differences between bank types, it seems reasonable to treat different bank types differently. Policy should especially avoid applying a regulation tailored to commercial banks that carry a systemic risk to the Euro zone to cooperative banks as well, that are neither as risky as commercial banks, nor similar in their organisation and business practices.

- It can be therefore argued, based on the present results, that the specificity of cooperative banks should be preserved, and that regulation aimed at reducing systemic risk ${ }^{9}$ should not necessarily apply to them (at least not in its current format), as their business practices already prevent them from carrying systemic risks.

- Finally, with reference to changes in the regulation particularly at the EU level, it can be suggested that the new regulations carrying increased compliance and personnel costs should be simplified for cooperative banks, or at least the burden of compliance costs should be eased.

It may be advisable for small cooperative banks in Italy (and elsewhere in Europe) to strengthen and thus render more effective the mutual support mechanisms and resolution schemes that are already provided by the cooperative credit network. This argument appears to be aligned with current policies designed by the Italian Government to consolidate the entire cooperative banking system. Conversely, it remains an open question whether these banks should apply for bail-in tools as suggested by the new EU directives (that came into force on January 1st, 2015, with the bail-in system to take effect from January 1st, 2016). Any regulation should account, as much as possible, both for the intended and unintended consequences. Specifically, the risks should be minimised that cooperative banks are forced to limit their ability to support local communities and economic agents, which, in turn, could lead to additional credit rationing, less credit being offered to local firms, and the hampering of new entrepreneurial activities.

\section{Acknowledgments}

This paper originates from my doctoral research "Corporate Governance and Bank Regulation: Evidence from the Italian Cooperative Banking System", discussed at the Faculty of Economics of the University of Ljubljana, Slovenia. I am grateful to Professor Aleksandra Gregorič for research supervision; to Professor Matej Marinč, Professor Marko Košak and Professor Francesco Pastore for useful feedbacks, and to Matteo Dimai for useful research assistance. I am grateful to the anonymous referee for valuable suggestions on how to improve the final version of paper. I am also indebted to the organisers of the $4^{\text {th }}$ EACB Academics and Stakeholders Day in Brussels and to the convenors of the stream "Employee Share Ownership in Central and Eastern Europe before, during, and after transformation: Some implications for participatory ways of organizing?" at the $9^{\text {th }}$ International Conference in Critical Management Studies at the University of Leicester, 8-10 $0^{\text {th }}$ July 2015, Dr. Olaf Kranz and Prof. Dr. Thomas Steger, for enabling me to present and discuss my paper there. The usual disclaimer applies.

\section{Literature}

Aiello, F., \& Bonanno, G. (2013). Profit and cost efficiency in the Italian banking industry (2006-2011). Economics and Business Letters, 2(4), 190-205, http://dx.doi. org/10.17811/ebl.2.4.2013.190-205

Angelini, P., \& Cetorelli, N. (2003). The Effects of Regulatory Reform on Competition in the Banking Industry. Journal of Money, Credit and Banking, 35(5), 663-684, http://dx.doi.org/10.17811/ebl.2.4.2013.190$\underline{205}$

Bank of England (2009). The Role of Macroprudential Policy. Discussion Paper November 2009.

Bank of International Settlements (2010). Principles for Enhancing Corporate Governance. Basel: Bank for International Settlements Communications.

Battistin, E., Graziano, C., \& Parigi, B. (2006). Connections or Performance: What Determines Turnover of Italian Bankers? WP 05-06-eco Dipartimento di Scienze Economiche. Udine: Università degli Studi di Udine, http://dx.doi.org/10.2139/ssrn.966429

Battistin, E., Graziano, C., \& Parigi, B. (2012). Connection and performance in bankers' turnover. European Economic Review, 56(3), 470-487, http://dx.doi. org/10.1016/j.euroecorev.2011.11.006

Bonanno, G. (2012). L'efficienza del sistema bancario italiano dal 2006 al 2010. Un'applicazione delle frontiere stocastiche. MPRA WP 46684.

Boot, A.W.A. (1999). Relationship Banking: What Do We Know? Journal of Financial Intermediation, 9, 7-25, http://dx.doi.org/10.1006/jfin.2000.0282

Boot, A.W.A., \& Thakor, A.V. (2000). Can Relationship Banking Survive Competition? Journal of Finance,

\footnotetext{
${ }^{9}$ Basel 3 advocates a framework to reduce the risks posed by 'systemically important financial institutions'. The new framework contains measures addressing the reduction of the cyclical effects of Basel 2 together with the reduction of systemic risk. Nevertheless, the new rules are not free from criticism. See the documents provided by the Bank of International Settlements (2010; http:// www.bis.org/bcbs/basel3.htm); guidelines and comments by PWC, 2011; and insightful comments available online, such as http:// www.risk.net/credit/analysis/1936514/basel-iii-tackles-systemic-risk-counterparty-risk
} 
55(2), 679-713, http://dx.doi.org/10.1111/00221082.00223

Cesarini, F. (2003). Il rapporto banca-impresa. Impresa, risparmio e intermediazione finanziaria: aspetti economici e giuridici, October, Trieste.

Chiaramonte, L., Poli, F. \& Oriani, M. (2013). Are Cooperative Banks a Lever for Promoting Bank Stability? Evidence from the Recent Financial Crisis in OECD Countries. European Financial Management, 21(3), 491-523, http://dx.doi.org/10.1111/j.1468036x.2013.12026.x

De Bonis, R. (2008). La banca. Rome: Carocci.

Dewatripont, M., \& Freixas, X. (2012). The Crisis Aftermath: New Regulatory Paradigms. London: CEPR

Di Salvo, R., Lopez, J. S., \& Pezzotta, A. (2004). L'evoluzione del relationship banking nei mercati creditizi locali: il ruolo delle Banche di Credito Cooperativo. Cooperazione al Credito, January-June, 81-113.

Dietrich, A. \& Wanzenried, G. (2009). What determines the profitability of commercial banks? New evidence from Switzerland. IFZ WP. 10/2009.

Dietrich, A. \& Wanzenried, G. (2011). Determinants of bank profitability before and during the crisis: evidence from Switzerland. Journal of International Financial Markets, Institutions and Money, 21(3), 307-327, http://dx.doi.org/10.1016/j.intfin.2010.11.002

EACB (2010). European Co-Operative Banks in the Financial and Economic Turmoil: First Assessment. Brussels: EACB Secretariat.

Fahlenbrach, R., Prilmeier, R., \&. Stulz, R.M. (2012). This Time is the Same: Using Bank Performance in 1998 to Explain Bank Performance during the Recent Financial Crisis. Journal of Finance, 67(6), 2139-2185, http:// dx.doi.org/10.1111/j.1540-6261.2012.01783.x

Ferraresi, M., Nordi, F., \& Rizzo, L. (2016). Una soluzione provinciale per le banche di credito cooperativo. Lavoce.info, 26 January 2016.

Ferri, G. (2008). Why Cooperative Banks Are Particularly Important in a Time of Credit Crunch (Mimeo). Bari: Università di Bari.

Ferri, G., Kalmi, P., \& Kerola, E. (2010). Organizational Structure and Performance in European Banks: A Reassessment. Paper presented at the conference "Financial Co-operative Approaches to Local Development Through Sustainable Innovation",

University of Trento, 10-11 June 2010 (Conference Proceedings).

Finocchiaro, A. (2007). Il network delle banche di credito cooperativo. Studi economici e sociali, 3, 11-22.

Freixas, X. (2009). Monetary Policy in a Systemic Crisis. Oxford Review of Economic Policy, 25(4), 630-653, http://dx.doi.org/10.2139/ssrn.1503349

Gallo, M., Graziano, M., \& Venturini, A. (2011). Gli effetti delle operazioni di concentrazione sulla crescita delle piccoli banche locali nel Nord Est. L'Economia del Nord Est (Bank of Italy proceedings), 441-479.

Girardone, C., Molyneux, P., \& Gardener Edvard, P. M. (2004). Analysing the Determinants of Bank Efficiency: The Case of Italian Banks. Applied Economics, 36, 215-227, http://dx.doi.org/10.1080/00036840420001 $\underline{75334}$

Goddard, J., Molyneux, P., \& Wilson, J.O. (2004). The Profitability of European Banks: A Cross-sectional and Dynamic Panel Analysis. The Manchester School, 72(3), 363-381, http://dx.doi.org/10.1111/j.14679957.2004.00397.x

Groeneveld, H. (2015). European Co-Operative Banking: Actual and Factual Assessment. Tilburg: TIAS.

Gutierrez, E. (2008). The Reform of Italian Cooperative Banks: Discussion of Proposals. IMF Working Paper WP/08/84, http://dx.doi. org/10.5089/9781451869361.001

Hesse, H., \& Cihak, M. (2007). Cooperative Banks and Financial Stability. IMF Working Paper WP/07/2, http://dx.doi.org/10.5089/9781451865660.001

Ianotta, G., Nocera, G., \& Sironi, A. (2007). Ownership Structure, Risk and Performance in the European Banking Industry. Journal of Banking and Finance, 31(7), 2127-2149, http://dx.doi.org/10.1016/j. jbankfin.2006.07.013

Il Sole 24 Ore (2015). All you need to know about Italian cooperative banks (BCCs). 10 June 2015.

IMF (2013). Italy: Financial System Stability Assessment. IMF Country Report 13/300. Washington: International Monetary Fund, http://dx.doi. org/10.5089/9781484311516.002

Karr, J. (2005). Performance Measure in Banking: Beyond ROE. Journal of Performance Management, 18(3), 56-70.

Leaven, L. \& Levine, R. (2006). Complex Ownership Structures and Corporate Valuations. NBER WP 12675, http://dx.doi.org/10.3386/w12675

Leonardi, A. (2009). Il credito cooperativo nella débâcle del sistema bancario italiano. Rivista della Cooperazione, 2, 152-172.

Lins, K.V., Volpin, P., \& Wagner, H.W. (2013). Does Family Control Matter? International Evidence from the 2008-2009 Financial Crisis. Review of Financial Studies, 26(10), 2583-2619, http://dx.doi.org/10.2139/ ssrn.1964764

Manetti, G., \& Bagnoli, L. (2013). Mutual and social efficiency of Italian co-operative banks: an empirical analysis. Annals of Public and Cooperative Economics, 84(3), 289-308, http://dx.doi.org/10.1111/apce.12015

Mattarocci, G., \& Gibilaro, L. (2008). Characteristics of the Recovery Process for Small Financial Intermediaries: The Case of Italian Cooperative Banks. Proceedings of the Academy of Banking Studies, 8(2) 
Mohsni, S., \& Otchere, I. (2015). Financial crisis, liquidity infusion and risk-taking: The case of Canadian banks. Journal of Banking Regulation, 16, 146-167, http:// dx.doi.org/10.1057/jbr.2014.2

Morgan Stanley Europe (2012). Italian Banks (February 6, 2012). London: Morgan Stanley Research.

Oliver Wyman (2014). Cooperative Banking: Leveraging the Cooperative Difference to Adapt to a New Environment. New York: Oliver Wyman.

PWC (2011). Basel 3 and Beyond: Systemically Important Financial Institutions. London: PricewaterhouseCoopers International Limited.

Quagliariello, M. (2008). Does Macroeconomy Affect Bank Stability? Journal of Banking Regulation, 9(2), 102-105, http://dx.doi.org/10.1057/jbr.2008.4

Rasmusen, E. (1988). Stock Banks and Mutual Banks. Journal of Law and Economics, 31, 395-422, http:// dx.doi.org/10.1086/467162

Stefancic, M. (2011). Cooperative Credit Network: Advantages and Challenges in Italian Cooperative Credit Banks. Euricse Working Paper 016/11, http:// dx.doi.org/10.2139/ssrn.1831078

Stefancic, M. (2014). Banke, finančna kriza in razprava o primernejši regulativi. Pravna Praksa, 2 (October 2014), 17-18.

Stefancic, M. (2014). Co-operative Banks in Italy. Review of International Co-operation, 6-16.

Stefancic, M. (2015). Alcuni spunti sull'abrogazione del voto capitario nelle banche popolari. nelmerito.com. 18 May 2015.

The Economist (2015). Not so popolari: Reform of Italy's biggest cooperative banks will help the sector to consolidate. 23 January 2015

Tidona Comunicazione (2013). Numero di Banche in Italia: 2000-2012. Milano: Tidona Comunicazione.

Tumpel-Gugerell, G.. (2005). Regulation, Competition and Integration in EU Banking: What Drives Performance? - Revisiting Freiburg. Freiburg: Faculty of Economics and Behavioural Sciences at the AlbertLudwigs University.

Vallascas, F., \& Hagendorff, J. (2013). The Risk Sensitivity of Capital Requirements: Evidence from an International Sample of Large Banks. Review of Finance, 17(6), 1947-1988, http://dx.doi.org/10.1093/ rof/rfs042

Wintoki, M.B., Link, J.S., \& Netter, J.M. (2012). Endogeneity and the dynamics of internal corporate governance. Journal of Financial Economics, 105, 581-606, $\quad$ http://dx.doi.org/10.1016/j. jfineco.2012.03.005

Mitja Stefancic holds a PhD in "Economics and Business" from the University of Ljubljana, Slovenia; an MPhil in "Modern Society and Global Transformations" from the University of Cambridge, UK; and a BA in Social Sciences from the University of Essex, UK. In the past he has been employed both in research institutes and in financial organisations such as the Banca di Credito Cooperativo del Carso based in Trieste, Italy. His research interests include financial regulation; democratic governance of firms; pluralism in market economies; origins of failures in contemporary markets; policies to contrast unemployment. 


\section{Appendix}

In this section some additional results are provided in support of the conclusions presented above, to check whether they are influenced by different specifications of the variables. In Table 7, the fixed effects regressions are replicated for the entire sample (this including banche popolari) with two modifications: 1 ) the start of the crisis is now defined as the year 2008, meaning that the crisis dummy now identifies the 2008-2012 period (CRISIS2008) and the full set of time dummy variables is showed using year 2005 as the reference year; 2) the cooperative and people's banks are considered together, defined by a new dummy variable COOPERATIVE. The models differ with regards to the dependent variable and the method used to cluster our standard errors (either by bank type or at the bank level). Moreover, the last two models in Table 7 use the newly defined dummy for cooperative banks (COOPERATIVE). All in all, the results presented in Table 7 re-confirm the conclusions reached in the main analysis. For ROAA, the coefficient of the COOP*CRISIS2008 interaction term (0.077) are very close to the ones of the fixed effects models in the main analysis (0.064 including popular banks and 0.083 excluding them), the coefficient is not significant, therefore one can conclude that cooperative credit banks have not had lower ROAA than commercial banks. No noteworthy differences are observable in the coefficients for the other variables.

For cost-to-income ratio, the results presented in Table 7 show a higher, significant coefficient for the logarithm of total assets, a lower coefficient for customer deposits on total assets, which is significant only when errors are clustered at bank level, a lower and not significant, but still positive, coefficient for liquid assets on customer \& short-term funding. Non interest income on revenues has a similar coefficient to the main analysis, but in Table 7 it is significant also when the errors are clustered at the bank type level. The coefficient for net loans on assets changes sign, becoming negative, but remains not significant. The same applies to the COOP*CRISIS2008 interaction term, so it can be concluded that there has been no significant difference between the impact of the crisis on the cost-toincome ratio of commercial and cooperative credit banks regardless of whether one sets the first crisis year to 2008 or 2009.

Merging people's banks into cooperative banks has little or no effect on the estimates. The only noticeable change is in the coefficient of the interaction term COOPERATIVE*CRISIS2008 with regards to cost-to-income ratio, that changes sign and becomes positive again (as in the main analysis). It should be noted, though, that it is still small and not significantly different from zero. Finally, the variable capturing the structure of banks' assets, i.e. the net value of loans in total bank assets (in percentage) is replaced with an alternative measure, namely the gross value of bank loans in the total bank assets. Again, the conclusions related to the main variable of interest remain unchanged. No significant changes in other coefficients can be reported. Gross loans on total assets have a significant negative effect on both cost-to-income ratio and loan quality. The results for fixed effects regression using the $C O O P$ dummy and new definition of the crisis (CRISIS2008) are presented in Table 8 . 
Table 7: Fixed effects regression for bank performance, cost efficiency and loan quality (Source: Author's calculations)

\begin{tabular}{|c|c|c|c|c|c|c|c|c|c|}
\hline & Bank type & Bank & Bank type & Bank & Bank type & Bank & \multicolumn{3}{|c|}{ Bank type/new definition of cooperative } \\
\hline VARIABLES & \multicolumn{2}{|c|}{ ROAA (v1) } & \multicolumn{2}{|c|}{$\begin{array}{l}\text { COST_EFFICIENCY } \\
\text { (v2) }\end{array}$} & \multicolumn{2}{|c|}{$\begin{array}{c}\text { LOAN_PROVISIONS } \\
(\mathrm{v} 3)\end{array}$} & v1 & v2 & v3 \\
\hline \multirow{2}{*}{ log total assets } & -0.080 & -0.080 & $-12.672 * *$ & $-12.672 * * *$ & $-0.331 *$ & $-0.331 * * *$ & -0.080 & $-12.656 * * *$ & $-0.331 * * *$ \\
\hline & {$[-0.203]$} & {$[-0.265]$} & {$[-4.157]$} & {$[-4.648]$} & {$[-2.396]$} & {$[-3.555]$} & {$[-0.265]$} & {$[-4.641]$} & {$[-3.580]$} \\
\hline \multirow[t]{2}{*}{$\begin{array}{l}\text { customer deposits on } \\
\text { assets }\end{array}$} & -0.001 & -0.001 & 0.138 & $0.138^{* * *}$ & -0.003 & -0.003 & -0.001 & $0.140^{* * *}$ & -0.003 \\
\hline & {$[-0.543]$} & {$[-0.158]$} & {$[1.858]$} & {$[2.629]$} & {$[-1.098]$} & {$[-1.064]$} & {$[-0.133]$} & {$[2.670]$} & {$[-1.133]$} \\
\hline \multirow[t]{2}{*}{$\begin{array}{l}\text { liquid assets on cus- } \\
\text { tomer s.t. funding }\end{array}$} & -0.005 & -0.005 & 0.019 & 0.019 & -0.000 & -0.000 & -0.005 & 0.018 & -0.000 \\
\hline & {$[-1.577]$} & [-1.449] & {$[0.761]$} & {$[0.343]$} & {$[-0.044]$} & {$[-0.057]$} & {$[-1.446]$} & {$[0.330]$} & {$[-0.062]$} \\
\hline \multirow[t]{2}{*}{$\begin{array}{l}\text { non interest income } \\
\text { on revenues }\end{array}$} & 0.007 & $0.007^{* *}$ & $-0.242^{*}$ & $-0.242 * * *$ & 0.001 & 0.001 & $0.007 * *$ & $-0.243 * * *$ & 0.001 \\
\hline & [1.639] & [2.377] & {$[-2.411]$} & {$[-4.408]$} & {$[0.838]$} & {$[0.627]$} & [2.310] & {$[-4.430]$} & [0.683] \\
\hline \multirow[t]{2}{*}{ net loans on assets } & 0.006 & $0.006^{*}$ & -0.081 & -0.081 & $-0.013 *$ & $-0.013 * * *$ & $0.006^{*}$ & -0.081 & $-0.012 * * *$ \\
\hline & {$[0.786]$} & {$[1.916]$} & {$[-1.461]$} & {$[-1.543]$} & {$[-2.890]$} & {$[-4.915]$} & {$[1.885]$} & {$[-1.548]$} & {$[-4.872]$} \\
\hline \multirow[t]{2}{*}{ equity on assets } & $0.020 * *$ & 0.020 & -0.072 & -0.072 & -0.041 & $-0.041 * * *$ & 0.020 & -0.070 & $-0.040 * * *$ \\
\hline & {$[4.512]$} & {$[0.675]$} & {$[-1.204]$} & {$[-0.287]$} & {$[-1.754]$} & {$[-4.418]$} & {$[0.672]$} & {$[-0.279]$} & {$[-4.428]$} \\
\hline \multirow[t]{2}{*}{ COOP*CRISIS2008 } & 0.077 & 0.077 & -0.163 & -0.163 & $-0.076^{*}$ & $-0.076^{*}$ & & & \\
\hline & [1.293] & {$[0.973]$} & {$[-0.738]$} & {$[-0.158]$} & {$[-2.757]$} & {$[-1.721]$} & & & \\
\hline \multirow[t]{2}{*}{$\begin{array}{c}\text { COOPERA- } \\
\text { TIVE*CRISIS2008 }\end{array}$} & & & & & & & 0.102 & 0.148 & $-0.104 * *$ \\
\hline & & & & & & & [1.025] & {$[0.117]$} & {$[-2.021]$} \\
\hline \multirow[t]{2}{*}{2006} & $0.182 * *$ & $0.182 * * *$ & $-4.008^{*}$ & $-4.008 * * *$ & $0.053^{*}$ & $0.053^{*}$ & $0.182 * * *$ & $-4.015^{* * *}$ & $0.053^{*}$ \\
\hline & [4.311] & [6.238] & {$[-2.900]$} & {$[-8.503]$} & [2.421] & [1.814] & [6.259] & [-8.509] & [1.830] \\
\hline \multirow[t]{2}{*}{2007} & $0.239 * *$ & $0.239 * * *$ & $-5.657^{*}$ & $-5.657 * * *$ & $0.203 * *$ & $0.203 * * *$ & $0.238 * * *$ & $-5.667 * * *$ & $0.203 * * *$ \\
\hline & [4.933] & [4.155] & {$[-2.863]$} & {$[-8.311]$} & [5.765] & [6.379] & [4.169] & [-8.318] & [6.401] \\
\hline \multirow[t]{2}{*}{2008} & -0.007 & -0.007 & $-3.965^{*}$ & $-3.965 * * *$ & $0.434 * * *$ & $0.434 * * *$ & -0.033 & $-4.194 * * *$ & $0.462 * * *$ \\
\hline & {$[-0.176]$} & {$[-0.084]$} & {$[-2.421]$} & {$[-3.275]$} & [7.679] & [8.259] & {$[-0.319]$} & [-2.978] & [7.737] \\
\hline \multirow[t]{2}{*}{2009} & $-0.358 * *$ & $-0.358 * * *$ & $4.041 * * *$ & $4.041 * * *$ & $0.517 * * *$ & $0.517 * * *$ & $-0.383 * * *$ & $3.813 * *$ & $0.544 * * *$ \\
\hline & {$[-4.849]$} & {$[-3.784]$} & [5.890] & [2.685] & [43.415] & [9.688] & {$[-3.615]$} & {$[2.236]$} & [9.001] \\
\hline \multirow[t]{2}{*}{2010} & $-0.574 * * *$ & $-0.574 * * *$ & $7.946 * * *$ & $7.946 * * *$ & $0.555^{* * *}$ & $0.555^{* * *}$ & $-0.599 * * *$ & $7.717 * * *$ & $0.582 * * *$ \\
\hline & [-6.433] & {$[-5.870]$} & [9.910] & [5.024] & [20.424] & [9.699] & {$[-5.525]$} & [4.346] & [9.156] \\
\hline \multirow[t]{2}{*}{2011} & $-0.566^{* * *}$ & $-0.566 * * *$ & $5.040 * *$ & $5.040 * * *$ & $0.625^{* * *}$ & $0.625^{* * *}$ & $-0.591 * * *$ & $4.816^{* * *}$ & $0.652 * * *$ \\
\hline & {$[-12.664]$} & {$[-5.620]$} & [5.382] & [3.359] & [18.162] & [10.511] & {$[-5.191]$} & [2.819] & [9.931] \\
\hline \multirow[t]{2}{*}{2012} & $-0.540 * * *$ & $-0.540 * * *$ & -0.633 & -0.633 & $1.239 * * *$ & $1.239 * * *$ & $-0.564 * * *$ & -0.853 & $1.266^{* * *}$ \\
\hline & {$[-8.281]$} & {$[-4.682]$} & {$[-0.803]$} & {$[-0.375]$} & [32.463] & [16.421] & {$[-4.666]$} & {$[-0.457]$} & [15.765] \\
\hline Observations & 4,240 & 4,240 & 4,233 & 4,233 & 4,154 & 4,154 & 4,240 & 4,233 & 4,154 \\
\hline R-squared & 0.216 & 0.216 & 0.198 & 0.198 & 0.334 & 0.334 & 0.216 & 0.198 & 0.334 \\
\hline
\end{tabular}

Note: Robust t-statistics reported in the brackets. * ,**, *** denote the $10 \%, 5 \%$ and $1 \%$ significance respectively. All regressions include bank fixed effects. Constant not reported. 
Table 8: Fixed effects regression for bank performance, cost efficiency and loan quality (Source: Author's calculations)

\begin{tabular}{|c|c|c|c|c|c|c|}
\hline \multirow[b]{2}{*}{ Dependent variable } & \multicolumn{3}{|c|}{ bank level clustering } & \multicolumn{3}{|c|}{ bank type clustering } \\
\hline & ROAA & $\begin{array}{c}\text { COST_EFFICIEN- } \\
\text { CY }\end{array}$ & $\begin{array}{l}\text { LOAN_PROVI- } \\
\text { SIONS }\end{array}$ & ROAA & $\begin{array}{l}\text { COST_EFFI- } \\
\text { CIENCY }\end{array}$ & $\begin{array}{l}\text { LOAN_PROVI- } \\
\text { SIONS }\end{array}$ \\
\hline \multirow[t]{2}{*}{ log total assets } & -0.091 & $-12.736^{* * *}$ & $-0.310 * * *$ & -0.091 & $-12.736^{* *}$ & $-0.310^{*}$ \\
\hline & {$[-0.302]$} & {$[-4.643]$} & {$[-3.300]$} & {$[-0.238]$} & {$[-4.038]$} & {$[-2.637]$} \\
\hline \multirow[t]{2}{*}{ customer deposits on assets } & -0.001 & $0.140 * * *$ & -0.003 & -0.001 & 0.140 & -0.003 \\
\hline & {$[-0.126]$} & {$[2.657]$} & {$[-1.087]$} & {$[-0.459]$} & {$[1.916]$} & {$[-1.114]$} \\
\hline \multirow[t]{2}{*}{$\begin{array}{l}\text { liquid assets on customer } \\
\text { s.t. funding }\end{array}$} & $-0.007 * *$ & 0.013 & $0.004 *$ & -0.007 & 0.013 & 0.004 \\
\hline & {$[-2.141]$} & {$[0.230]$} & {$[1.826]$} & {$[-2.294]$} & {$[0.516]$} & {$[1.401]$} \\
\hline \multirow[t]{2}{*}{$\begin{array}{l}\text { non interest income on } \\
\text { revenues }\end{array}$} & $0.006^{* *}$ & $-0.243^{* * *}$ & 0.001 & 0.006 & $-0.243^{*}$ & 0.001 \\
\hline & {$[2.295]$} & {$[-4.415]$} & {$[0.811]$} & {$[1.607]$} & [-2.419] & [0.994] \\
\hline \multirow[t]{2}{*}{ gross loans on assets } & 0.001 & $-0.091 *$ & $-0.005^{*}$ & 0.001 & -0.091 & -0.005 \\
\hline & {$[0.308]$} & {$[-1.864]$} & {$[-1.904]$} & {$[0.141]$} & {$[-1.509]$} & {$[-1.573]$} \\
\hline \multirow[t]{2}{*}{ equity on assets } & 0.019 & -0.073 & $-0.040 * * *$ & $0.019 * *$ & -0.073 & -0.040 \\
\hline & {$[0.650]$} & {$[-0.289]$} & {$[-4.320]$} & {$[4.669]$} & {$[-1.277]$} & {$[-1.733]$} \\
\hline \multirow[t]{2}{*}{ COOP*CRISIS2008 } & 0.079 & -0.181 & $-0.079 *$ & 0.079 & -0.181 & $-0.079 *$ \\
\hline & {$[0.990]$} & {$[-0.175]$} & {$[-1.772]$} & {$[1.259]$} & {$[-0.766]$} & {$[-2.470]$} \\
\hline \multirow[t]{2}{*}{2006} & $0.188^{* * *}$ & $-3.888 * * *$ & 0.045 & $0.188^{* *}$ & $-3.888^{*}$ & $0.045^{*}$ \\
\hline & [6.019] & {$[-8.023]$} & {$[1.543]$} & [4.761] & {$[-3.073]$} & [2.434] \\
\hline \multirow[t]{2}{*}{2007} & $0.250 * * *$ & $-5.515 * * *$ & $0.186^{* * *}$ & $0.250^{* *}$ & $-5.515^{*}$ & $0.186^{* * *}$ \\
\hline & {$[4.225]$} & {$[-7.885]$} & [5.809] & [5.324] & [-2.989] & [6.829] \\
\hline \multirow[t]{2}{*}{2008} & 0.002 & $-3.789^{* * *}$ & $0.420 * * *$ & 0.002 & $-3.789 *$ & $0.420 * * *$ \\
\hline & {$[0.027]$} & {$[-3.075]$} & {$[8.125]$} & {$[0.055]$} & {$[-2.584]$} & [8.347] \\
\hline \multirow[t]{2}{*}{2009} & $-0.349 * * *$ & $4.238 * * *$ & $0.503 * * *$ & $-0.349 * *$ & $4.238^{* *}$ & $0.503 * * *$ \\
\hline & {$[-3.632]$} & {$[2.778]$} & {$[9.508]$} & {$[-4.571]$} & {$[5.412]$} & [29.835] \\
\hline \multirow[t]{2}{*}{2010} & $-0.561 * * *$ & $8.176^{* * *}$ & $0.536^{* * *}$ & $-0.561 * * *$ & $8.176^{* * *}$ & $0.536 * * *$ \\
\hline & {$[-5.653]$} & [5.105] & [9.352] & {$[-6.246]$} & {$[8.218]$} & [21.318] \\
\hline \multirow[t]{2}{*}{2011} & $-0.561 * * *$ & $5.295 * * *$ & $0.619^{* * *}$ & $-0.561 * * *$ & $5.295 * * *$ & $0.619 * * *$ \\
\hline & {$[-5.508]$} & {$[3.482]$} & [10.673] & {$[-11.431]$} & {$[7.261]$} & [23.168] \\
\hline \multirow[t]{2}{*}{2012} & $-0.557 * * *$ & -0.375 & $1.273 * * *$ & $-0.557 * * *$ & -0.375 & $1.273 * * *$ \\
\hline & {$[-4.796]$} & {$[-0.220]$} & [16.615] & {$[-7.647]$} & {$[-0.533]$} & [28.845] \\
\hline Observations & 4,240 & 4,233 & 4,154 & 4,240 & 4,233 & 4,154 \\
\hline R-squared & 0.214 & 0.198 & 0.325 & 0.214 & 0.198 & 0.325 \\
\hline
\end{tabular}

Note: Robust t-statistics reported in the brackets. $*, * *, * *$ denote the $10 \%, 5 \%$ and $1 \%$ significance respectively. All regressions include bank fixed effects. Constant not reported 